\title{
SYMMETRIC AND NON-SYMMETRIC VARIANTS OF NITSCHE'S METHOD FOR CONTACT PROBLEMS IN ELASTICITY: THEORY AND NUMERICAL EXPERIMENTS
}

\author{
FRANZ CHOULY, PATRICK HILD, AND YVES RENARD
}

\begin{abstract}
A general Nitsche method, which encompasses symmetric and non-symmetric variants, is proposed for frictionless unilateral contact problems in elasticity. The optimal convergence of the method is established both for two- and three-dimensional problems and Lagrange affine and quadratic finite element methods. Two- and three-dimensional numerical experiments illustrate the theory.
\end{abstract}

\section{INTRODUCTION}

In solid mechanics, the numerical implementation of contact and impact problems generally uses the Finite Element Method (FEM) (see [17, 18, 20, 27, 30, 39, 40,). The non-linear contact conditions involved on part of the boundary lead naturally to a variational inequality (see, e.g., [15]).

We consider in this paper a special FEM inspired from Nitsche's method 33] (see also [3] for an early extension to the Discontinuous Galerkin framework) in which the coercivity condition for the domain bilinear form $A_{\theta \gamma}$ (see (99)) is the same as in the standard formulation (see, e.g., [4]). This method aims at treating the boundary or interface conditions in a weak sense, thanks to a consistent penalty term. So it differs from standard penalization techniques which are typically non-consistent [27]. Moreover, unlike mixed methods (see, e.g., [20]), no additional unknown (Lagrange multiplier) is needed. Nitsche's method has been widely applied during these last years to problems involving linear conditions on the boundary of a domain or in the interface between sub-domains; see, e.g, . 36] for the Dirichlet problem or [4 for domain decomposition with non-matching meshes. More recently, in [19, 21] it has been adapted for bilateral (persistent) contact, which still involves linear boundary conditions on the contact zone (note that an algorithm for unilateral contact which involves Nitsche's method in its original form is given and implemented in [19]). An extension to large strain bilateral contact has been performed in [41.

In a previous work a symmetric Nitsche-based method has been proposed for (non-linear) unilateral contact conditions (see [9]). We established its optimal convergence in the $H^{1}(\Omega)$-norm of order $O\left(h^{\frac{1}{2}+\nu}\right)$ for a solution of regularity $H^{\frac{3}{2}+\nu}(\Omega)$ $\left(0<\nu \leq \frac{1}{2}\right)$. Furthermore, no additional assumption on the contact zone is needed for the proof, such as an increased regularity of the contact stress or a finite number of transitions between contact and non-contact. Also, the standard FEM for contact consists in a direct conforming approximation of the variational inequality,

Received by the editor January 14, 2013 and, in revised form, September 4, 2013.

2010 Mathematics Subject Classification. Primary 65N12, 65N30, 74M15.

Key words and phrases. Unilateral contact, finite elements, Nitsche's method. 
with the elastic displacement as the only unknown. For this standard FEM and also for all the other approaches such as mixed/hybrid methods (e.g., 6, 22, 29]), stabilized mixed methods (e.g., 24]), penalty methods (e.g., [10]), no such proof of optimal convergence has been established to the best of our knowledge where the solution $\mathbf{u}$ is in $H^{\frac{3}{2}+\nu}(\Omega)\left(0<\nu \leq \frac{1}{2}\right)$. We refer, e.g., to [25, 26] for recent reviews on a priori error estimates for contact problems.

In the present paper we introduce an extension of the symmetric method of $[9$, which allows non-symmetric variants, depending upon a new parameter called $\theta$. The symmetric case is recovered when $\theta=1$. In this new method the advantage of the symmetric formulation consisting in the positivity of the Nitsche penalty term is generally lost. Nevertheless this extension presents some other advantages, mostly from the numerical viewpoint. In particular, one of its variants $(\theta=0)$ involves a reduced quantity of terms, which makes it easier to implement and to extend to contact problems involving non-linear elasticity. Also, for $\theta=-1$, the wellposedness of the discrete formulation and the optimal convergence are preserved irrespectively of the value of the Nitsche parameter. For all the values of $\theta$, the optimal convergence rates can still be obtained. We prove this optimal convergence property and illustrate it on several numerical experiments. Note that the behavior of the generalized Newton algorithm when applied to this method has already been studied in 35, in the frictionless and frictional cases and for two values of $\theta$ ( 0 and 1 ). This study illustrated the better numerical performances of the non-symmetric variant $\theta=0$, which requires less Newton iterations to converge, for a wider range of the Nitsche parameter. Values of $\theta$ different from 0 and 1 have not been studied previously.

Our paper is outlined as follows. In Section 2, we recall the continuous (strong and weak) formulations for unilateral contact problems and introduce our Nitschebased FEM. In Section 3. we carry out the numerical analysis of this method: we prove its consistency, the existence and uniqueness of solutions, and at last its optimal convergence. Numerical experiments in 2D and 3D are described in Section 4. which illustrate the convergence properties for different values of the numerical parameters, and in particular $\theta$. In Section 5 conclusions are drawn and some perspectives are given.

Let us introduce some useful notation. In what follows, bold letters like $\mathbf{u}, \mathbf{v}$, indicate vector or tensor valued quantities, while the capital letters (e.g., V, K, etc.) represent functional sets involving vector fields. As usual, we denote by $\left(H^{s}(.)\right)^{d}$, $s \in \mathbb{R}, d=1,2,3$, the Sobolev spaces in one, two or three space dimensions (see [1]). The usual norm of $\left(H^{s}(D)\right)^{d}$ is denoted by $\|\cdot\|_{s, D}$ and we keep the same notation when $d=1$ or $d>1$. The letter $C$ stands for a generic constant, independent of the discretization parameters.

\section{SEtTing}

2.1. The unilateral contact problem. We consider an elastic body whose reference configuration is represented by the domain $\Omega$ in $\mathbb{R}^{d}$ with $d=2$ or $d=3$. Small strain assumptions are made, as well as plane strain when $d=2$. The boundary $\partial \Omega$ of $\Omega$ is polygonal or polyhedral and we suppose that $\partial \Omega$ consists in three nonoverlapping parts $\Gamma_{D}, \Gamma_{N}$ and the contact boundary $\Gamma_{C}$, with meas $\left(\Gamma_{D}\right)>0$ and meas $\left(\Gamma_{C}\right)>0$. The contact boundary is supposed to be a straight line segment when $d=2$ or a polygon when $d=3$ to simplify. The unit outward normal vector 
on $\partial \Omega$ is denoted $\mathbf{n}$. In its initial stage, the body is in contact on $\Gamma_{C}$ with a rigid foundation (the extension to two elastic bodies in contact can be easily made, at least for small strain models) and we suppose that the unknown final contact zone after deformation will be included in $\Gamma_{C}$. The body is clamped on $\Gamma_{D}$ for the sake of simplicity. It is subjected to volume forces $\mathbf{f} \in\left(L^{2}(\Omega)\right)^{d}$ and to surface loads $\mathbf{g} \in\left(L^{2}\left(\Gamma_{N}\right)\right)^{d}$.

The unilateral contact problem in linear elasticity consists in finding the displacement field $\mathbf{u}: \Omega \rightarrow \mathbb{R}^{d}$ verifying the equations and conditions (1)-(2):

$$
\begin{aligned}
\operatorname{div} \boldsymbol{\sigma}(\mathbf{u})+\mathbf{f} & =\mathbf{0} & & \text { in } \Omega, \\
\boldsymbol{\sigma}(\mathbf{u}) & =\mathbf{A} \varepsilon(\mathbf{u}) & & \text { in } \Omega, \\
\mathbf{u} & =\mathbf{0} & & \text { on } \Gamma_{D} \\
\boldsymbol{\sigma}(\mathbf{u}) \mathbf{n} & =\mathbf{g} & & \text { on } \Gamma_{N}
\end{aligned}
$$

where $\boldsymbol{\sigma}=\left(\sigma_{i j}\right), 1 \leq i, j \leq d$, stands for the stress tensor field and $\mathbf{d i v}$ denotes the divergence operator of tensor valued functions. The notation $\varepsilon(\mathbf{v})=\left(\boldsymbol{\nabla} \mathbf{v}+\boldsymbol{\nabla} \mathbf{v}^{T}\right) / 2$ represents the linearized strain tensor field and $\mathbf{A}$ is the fourth order symmetric elasticity tensor having the usual uniform ellipticity and boundedness property. For any displacement field $\mathbf{v}$ and for any density of surface forces $\boldsymbol{\sigma}(\mathbf{v}) \mathbf{n}$ defined on $\partial \Omega$ we adopt the notation

$$
\mathbf{v}=v_{n} \mathbf{n}+v_{\mathbf{t}} \quad \text { and } \quad \boldsymbol{\sigma}(\mathbf{v}) \mathbf{n}=\sigma_{n}(\mathbf{v}) \mathbf{n}+\sigma_{\mathbf{t}}(\mathbf{v})
$$

where $v_{\mathbf{t}}\left(\right.$ resp. $\left.\sigma_{\mathbf{t}}(\mathbf{v})\right)$ are the tangential components of $\mathbf{v}(\operatorname{resp} . \quad \boldsymbol{\sigma}(\mathbf{v}) \mathbf{n})$. The conditions describing unilateral contact without friction on $\Gamma_{C}$ are:

$$
\begin{aligned}
(\text { i }) & u_{n} & \leq 0, \\
\text { (ii) } & \sigma_{n}(\mathbf{u}) & \leq 0, \\
\text { (iii) } & \sigma_{n}(\mathbf{u}) u_{n} & =0, \\
\text { (iv }) & \sigma_{\mathbf{t}}(\mathbf{u}) & =0 .
\end{aligned}
$$

We introduce the Hilbert space $\mathbf{V}$ and the convex cone $\mathbf{K}$ of admissible displacements which satisfy the noninterpenetration on the contact zone $\Gamma_{C}$ :

$$
\mathbf{V}:=\left\{\mathbf{v} \in\left(H^{1}(\Omega)\right)^{d}: \mathbf{v}=\mathbf{0} \text { on } \Gamma_{D}\right\}, \quad \mathbf{K}:=\left\{\mathbf{v} \in \mathbf{V}: v_{n}=\mathbf{v} \cdot \mathbf{n} \leq 0 \text { on } \Gamma_{C}\right\} .
$$

We define also

$$
a(\mathbf{u}, \mathbf{v}):=\int_{\Omega} \boldsymbol{\sigma}(\mathbf{u}): \varepsilon(\mathbf{v}) d \Omega, \quad L(\mathbf{v}):=\int_{\Omega} \mathbf{f} \cdot \mathbf{v} d \Omega+\int_{\Gamma_{N}} \mathbf{g} \cdot \mathbf{v} d \Gamma,
$$

for any $\mathbf{u}$ and $\mathbf{v}$ in $\mathbf{V}$. From the previous assumptions, we deduce that $a(\cdot, \cdot)$ is bilinear, symmetric, $\mathbf{V}$-elliptic and continuous on $\mathbf{V} \times \mathbf{V}$. We see also that $L(\cdot)$ is a continuous linear form on $\mathbf{V}$. The weak formulation of Problem (11)-(2), as a variational inequality (see $[15,20,27]$ ), reads as:

$$
\left\{\begin{array}{l}
\text { Find } \mathbf{u} \in \mathbf{K} \text { such that: } \\
a(\mathbf{u}, \mathbf{v}-\mathbf{u}) \geq L(\mathbf{v}-\mathbf{u}), \quad \forall \mathbf{v} \in \mathbf{K} .
\end{array}\right.
$$

Stampacchia's Theorem ensures that problem (3) admits a unique solution. 
2.2. A general Nitsche-based finite element method. Let $\mathbf{V}^{h} \subset \mathbf{V}$ be a family of finite dimensional vector spaces (see $7,11,14]$ ) indexed by $h$ coming from a family $\mathcal{T}^{h}$ of triangulations of the domain $\Omega\left(h=\max _{T \in \mathcal{T}^{h}} h_{T}\right.$ where $h_{T}$ is the diameter of $T$ ). The family of triangulations is supposed regular (i.e., there exists $\sigma>0$ such that $\forall T \in \mathcal{T}^{h}, h_{T} / \rho_{T} \leq \sigma$ where $\rho_{T}$ denotes the radius of the inscribed ball in $T$ ) and conformal to the subdivision of the boundary into $\Gamma_{D}, \Gamma_{N}$ and $\Gamma_{C}$ (i.e., a face of an element $T \in \mathcal{T}^{h}$ is not allowed to have simultaneous non-empty intersection with more than one part of the subdivision). To fix ideas, we choose a standard Lagrange finite element method of degree $k$ with $k=1$ or $k=2$, i.e.:

$$
\mathbf{V}^{h}:=\left\{\mathbf{v}^{h} \in\left(\mathscr{C}^{0}(\bar{\Omega})\right)^{d}: \mathbf{v}_{\mid T}^{h} \in\left(P_{k}(T)\right)^{d}, \forall T \in \mathcal{T}^{h}, \mathbf{v}^{h}=\mathbf{0} \text { on } \Gamma_{D}\right\} .
$$

However, the analysis would be similar for any $\mathscr{C}^{0}$-conforming finite element method.

Let us introduce the notation $[\cdot]_{+}$for the positive part of a scalar quantity $a \in \mathbb{R}$ :

$$
[a]_{+}:=\left\{\begin{array}{rr}
a & \text { if } a>0 \\
0 & \text { otherwise }
\end{array}\right.
$$

For the positive part satisfying

$$
a \leq[a]_{+}, \quad a[a]_{+}=[a]_{+}^{2}, \quad \forall a \in \mathbb{R},
$$

we can deduce the following monotonicity property:

$$
\begin{aligned}
\left([a]_{+}-[b]_{+}\right)(a-b) & =a[a]_{+}+b[b]_{+}-b[a]_{+}-a[b]_{+} \\
& \geq[a]_{+}^{2}+[b]_{+}^{2}-2[a]_{+}[b]_{+} \\
& =\left([a]_{+}-[b]_{+}\right)^{2} \geq 0 .
\end{aligned}
$$

Note that conditions (5) and (6) can be straightforwardly extended to real-valued functions.

The derivation of a Nitsche-based method comes from a classical reformulation (see for instance [2]) of the contact conditions (2) (i)-(iii), for a given $\gamma>0$ :

$$
\sigma_{n}(\mathbf{u})=-\frac{1}{\gamma}\left[u_{n}-\gamma \sigma_{n}(\mathbf{u})\right]_{+} \cdot
$$

Remark 2.1. Note that condition (7) is still equivalent to (2) (i)-(iii) on $\Gamma_{C}$ when $\gamma$ is a positive function defined on $\Gamma_{C}$ instead of a positive constant.

Now let $\theta \in \mathbb{R}$ be a fixed parameter, and let $\mathbf{u}$ be the solution of the unilateral contact problem in its strong form (1)-(2). We assume that $\mathbf{u}$ is sufficiently regular so that all the following calculations make sense. From the Green formula, equations (11) and (2) (iv), we get for every $\mathbf{v} \in \mathbf{V}$ :

$$
a(\mathbf{u}, \mathbf{v})-\int_{\Gamma_{C}} \sigma_{n}(\mathbf{u}) v_{n} d \Gamma=L(\mathbf{v}) .
$$

With the splitting $v_{n}=\left(v_{n}-\theta \gamma \sigma_{n}(\mathbf{v})\right)+\theta \gamma \sigma_{n}(\mathbf{v})$, we obtain

$$
a(\mathbf{u}, \mathbf{v})-\int_{\Gamma_{C}} \theta \gamma \sigma_{n}(\mathbf{u}) \sigma_{n}(\mathbf{v}) d \Gamma-\int_{\Gamma_{C}} \sigma_{n}(\mathbf{u})\left(v_{n}-\theta \gamma \sigma_{n}(\mathbf{v})\right) d \Gamma=L(\mathbf{v}) .
$$

Finally, using condition (7), we still have for every $\mathbf{v} \in \mathbf{V}$ :

(8) $a(\mathbf{u}, \mathbf{v})-\int_{\Gamma_{C}} \theta \gamma \sigma_{n}(\mathbf{u}) \sigma_{n}(\mathbf{v}) d \Gamma+\int_{\Gamma_{C}} \frac{1}{\gamma}\left[u_{n}-\gamma \sigma_{n}(\mathbf{u})\right]_{+}\left(v_{n}-\theta \gamma \sigma_{n}(\mathbf{v})\right) d \Gamma=L(\mathbf{v})$. 
Formula (8) is the starting point of our Nitsche-based formulation. We remark that it may have no sense at the continuous level if $\mathbf{u}$ lacks of regularity (the only assumption $\mathbf{u} \in \mathbf{V}$ is not sufficient to justify the above calculations). Nevertheless, and as in the stabilized Lagrange multiplier method 24, we consider in what follows that $\gamma$ is a positive piecewise constant function on the contact interface $\Gamma_{C}$ defined for any $\mathbf{x} \in \Gamma_{C}$ lying on the relative interior of $\Gamma_{C} \cap T$ for a (closed) element $T$ having a non-empty intersection of dimension $d-1$ with $\Gamma_{C}$ by

$$
\gamma(\mathbf{x})=\gamma_{0} h_{T}
$$

where $\gamma_{0}$ is a positive given constant (the value of $\gamma$ on element intersections has no influence). This allows us to define a discrete counterpart of (8). Let us introduce for this purpose the discrete linear operator

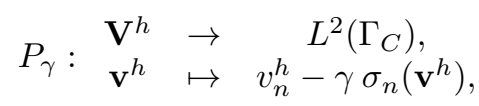

and also the bilinear form

$$
A_{\theta \gamma}\left(\mathbf{u}^{h}, \mathbf{v}^{h}\right):=a\left(\mathbf{u}^{h}, \mathbf{v}^{h}\right)-\int_{\Gamma_{C}} \theta \gamma \sigma_{n}\left(\mathbf{u}^{h}\right) \sigma_{n}\left(\mathbf{v}^{h}\right) d \Gamma .
$$

Remark 2.2. With the previous notations, we see that problem (3) could be formally written as follows:

$\left\{\begin{array}{l}\text { Find a sufficiently regular } \mathbf{u} \in \mathbf{V} \text { such that: } \\ A_{\theta \gamma}(\mathbf{u}, \mathbf{v})+\int_{\Gamma_{C}} \frac{1}{\gamma}\left[P_{\gamma}(\mathbf{u})\right]_{+} P_{\theta \gamma}(\mathbf{v}) d \Gamma=L(\mathbf{v}), \text { for all sufficiently regular } \mathbf{v} \in \mathbf{V},\end{array}\right.$

with $P_{\theta \gamma}(\mathbf{v}):=v_{n}-\theta \gamma \sigma_{n}(\mathbf{v})$. Note in particular that $P_{\gamma}(\mathbf{u})$ and $P_{\theta \gamma}(\mathbf{u})$ are welldefined and belong to $L^{2}\left(\Gamma_{C}\right)$ provided that $\mathbf{u} \in\left(H^{\frac{3}{2}+\nu}(\Omega)\right)^{d}(\nu>0)$.

Our generalized Nitsche-based method then reads:

$$
\left\{\begin{array}{l}
\text { Find } \mathbf{u}^{h} \in \mathbf{V}^{h} \text { such that: } \\
A_{\theta \gamma}\left(\mathbf{u}^{h}, \mathbf{v}^{h}\right)+\int_{\Gamma_{C}} \frac{1}{\gamma}\left[P_{\gamma}\left(\mathbf{u}^{h}\right)\right]_{+} P_{\theta \gamma}\left(\mathbf{v}^{h}\right) d \Gamma=L\left(\mathbf{v}^{h}\right), \quad \forall \mathbf{v}^{h} \in \mathbf{V}^{h}
\end{array}\right.
$$

Remark 2.3. The introduction of an additional numerical parameter $\theta$ allows us to introduce some new interesting variants acting on the symmetry / skew-symmetry / non-symmetry of the discrete formulation. Moreover, a unified analysis of all these variants can be performed. Note that some values of $\theta$ may be of special interest:

- for $\theta=1$ we recover the symmetric method proposed and analyzed in 9 ,

- for $\theta=0$ we recover a very simple non-symmetric version close to the classical penalty method,

- for $\theta=-1$ we obtain a skew-symmetric version which has the remarkable property to be well-posed and convergent irrespectively of the value of $\gamma_{0}>$ 0 (see Theorem 3.4 and Theorem 3.8). 
Remark 2.4. As in the symmetric case [9], we can rewrite the formulation (10) in a mixed form as follows:

$$
\left\{\begin{array}{l}
\text { Find }\left(\mathbf{u}^{h}, \lambda^{h}\right) \in \mathbf{V}^{h} \times L_{-}^{2}\left(\Gamma_{C}\right) \text { such that: } \\
a\left(\mathbf{u}^{h}, \mathbf{v}^{h}\right)-\int_{\Gamma_{C}} \lambda^{h} v_{n}^{h} d \Gamma+\int_{\Gamma_{C}} \theta \gamma\left(\lambda^{h}-\sigma_{n}\left(\mathbf{u}^{h}\right)\right) \sigma_{n}\left(\mathbf{v}^{h}\right) d \Gamma=L\left(\mathbf{v}^{h}\right), \quad \forall \mathbf{v}^{h} \in \mathbf{V}^{h}, \\
\int_{\Gamma_{C}}\left(\mu-\lambda^{h}\right) u_{n}^{h} d \Gamma+\int_{\Gamma_{C}} \gamma\left(\mu-\lambda^{h}\right)\left(\lambda^{h}-\sigma_{n}\left(\mathbf{u}^{h}\right)\right) d \Gamma \geq 0, \quad \forall \mu \in L_{-}^{2}\left(\Gamma_{C}\right),
\end{array}\right.
$$

where $L_{-}^{2}\left(\Gamma_{C}\right):=\left\{\mu \in L^{2}\left(\Gamma_{C}\right) \mid \mu \leq 0\right.$ a.e. on $\left.\Gamma_{C}\right\}$ and with $\lambda^{h}=-\frac{1}{\gamma}\left[P_{\gamma}\left(\mathbf{u}^{h}\right)\right]_{+}$. Note that formally, in the case $\theta \neq 1$, this mixed form is somehow different from the stabilized method applied to unilateral contact (see 24]). In particular the stabilization term in the first equation vanishes when $\theta=0$.

\section{Analysis of the Nitsche-BAsed method}

In this section, we carry out the mathematical analysis of the method (10). A difference between Nitsche's method and classical penalty methods [10, 27 is the property of consistency, which we first show in \$3.1 The proof of the well-posedness of the (non-linear) discrete problem (10) is carried out in 3.2 The error analysis is finally detailed in 33.3 . We show that the method converges in an optimal way when the mesh size $h$ vanishes.

3.1. Consistency. Like Nitsche's method for second order elliptic problems with Dirichlet boundary conditions or domain decomposition [4, our Nitsche-based formulation (10) for unilateral contact is consistent:

Lemma 3.1. The Nitsche-based method for contact is consistent: suppose that the solution $\mathbf{u}$ of (11) (2) lies in $\left(H^{\frac{3}{2}+\nu}(\Omega)\right)^{d}$ with $\nu>0$ and $d=2,3$. Then $\mathbf{u}$ is also the solution of

$$
A_{\theta \gamma}\left(\mathbf{u}, \mathbf{v}^{h}\right)+\int_{\Gamma_{C}} \frac{1}{\gamma}\left[P_{\gamma}(\mathbf{u})\right]_{+} P_{\theta \gamma}\left(\mathbf{v}^{h}\right) d \Gamma=L\left(\mathbf{v}^{h}\right), \quad \forall \mathbf{v}^{h} \in \mathbf{V}^{h} .
$$

Proof. Let $\mathbf{u}$ be the solution of (1)-(2) and set $\mathbf{v}^{h} \in \mathbf{V}^{h}$. Since $\mathbf{u} \in\left(H^{\frac{3}{2}+\nu}(\Omega)\right)^{d}$ and $\nu>0$, we have $\sigma_{n}(\mathbf{u}) \in H^{\nu}\left(\Gamma_{C}\right) \subset L^{2}\left(\Gamma_{C}\right)$. As a result, $A_{\theta \gamma}\left(\mathbf{u}, \mathbf{v}^{h}\right)$ makes sense and $P_{\gamma}(\mathbf{u}) \in L^{2}\left(\Gamma_{C}\right)$. On the one hand, we use the definition of $P_{\gamma}$, of $A_{\theta \gamma}(\cdot, \cdot)$ and the reformulation (7) of the contact conditions to obtain:

$$
\begin{aligned}
& A_{\theta \gamma}\left(\mathbf{u}, \mathbf{v}^{h}\right)+\int_{\Gamma_{C}} \frac{1}{\gamma}\left[P_{\gamma}(\mathbf{u})\right]_{+} P_{\theta \gamma}\left(\mathbf{v}^{h}\right) d \Gamma \\
= & a\left(\mathbf{u}, \mathbf{v}^{h}\right)-\int_{\Gamma_{C}} \theta \gamma \sigma_{n}(\mathbf{u}) \sigma_{n}\left(\mathbf{v}^{h}\right) d \Gamma+\int_{\Gamma_{C}} \frac{1}{\gamma}\left(-\gamma \sigma_{n}(\mathbf{u})\right)\left(v_{n}^{h}-\theta \gamma \sigma_{n}\left(\mathbf{v}^{h}\right)\right) d \Gamma \\
= & a\left(\mathbf{u}, \mathbf{v}^{h}\right)-\int_{\Gamma_{C}} \sigma_{n}(\mathbf{u}) v_{n}^{h} d \Gamma .
\end{aligned}
$$

On the other hand, with equations (11)-(2) and an integration by parts, we get

$$
a\left(\mathbf{u}, \mathbf{v}^{h}\right)-\int_{\Gamma_{C}} \sigma_{n}(\mathbf{u}) v_{n}^{h} d \Gamma=L\left(\mathbf{v}^{h}\right),
$$

which ends the proof. 
3.2. Well-posedness. To prove well-posedness of our Nitsche-based formulation, we first need the following classical property.

Lemma 3.2. There exists $C>0$, independent of the parameter $\gamma_{0}$ and of the mesh size $h$, such that

$$
\left\|\gamma^{\frac{1}{2}} \sigma_{n}\left(\mathbf{v}^{h}\right)\right\|_{0, \Gamma_{C}}^{2} \leq C \gamma_{0}\left\|\mathbf{v}^{h}\right\|_{1, \Omega}^{2}
$$

for all $\mathbf{v}^{h} \in \mathbf{V}^{h}$.

Proof. It follows from the definition of $\sigma_{n}\left(\mathbf{v}^{h}\right)$ and the boundedness of $\mathbf{A}$ that

$$
\left\|\gamma^{\frac{1}{2}} \sigma_{n}\left(\mathbf{v}^{h}\right)\right\|_{0, \Gamma_{C}}^{2} \leq \gamma_{0} h\left\|\sigma_{n}\left(\mathbf{v}^{h}\right)\right\|_{0, \Gamma_{C}}^{2} \leq C \gamma_{0} h\left\|\nabla \mathbf{v}^{h}\right\|_{0, \Gamma_{C}}^{2} .
$$

Then estimation (11) is obtained using a scaling argument; see [37, Lemma 2.1, p. 24] for a detailed proof in the general case (for an arbitrary degree $k$ and any dimension $d$ ).

Remark 3.3. Note that contrary to the penalty case or to the symmetric case of Nitsche's method (i.e., $\theta=1$ ), the integral term on $\Gamma_{C}$,

$$
\int_{\Gamma_{C}} \frac{1}{\gamma}\left[P_{\gamma}\left(\mathbf{u}^{h}\right)\right]_{+} P_{\theta \gamma}\left(\mathbf{v}^{h}\right)
$$

is not necessarily non-negative when $\mathbf{v}^{h}=\mathbf{u}^{h}$ and $\theta \neq 1$.

We then show that problem (10) is well-posed using an argument by Brezis for M-type and pseudo-monotone operators 8] (see also [31 and 28]).

Theorem 3.4. Suppose that one of the two following assumptions hold:

(1) $\theta \neq-1$ and $\gamma_{0}>0$ is sufficiently small,

(2) $\theta=-1$ and $\gamma_{0}>0$.

Then problem (10) admits one unique solution $\mathbf{u}^{h}$ in $\mathbf{V}^{h}$.

Proof. Using the Riesz representation theorem, we define a (non-linear) operator $\mathbf{B}^{h}: \mathbf{V}^{h} \rightarrow \mathbf{V}^{h}$, by means of the formula

$$
\left(\mathbf{B}^{h} \mathbf{v}^{h}, \mathbf{w}^{h}\right)_{1, \Omega}:=A_{\theta \gamma}\left(\mathbf{v}^{h}, \mathbf{w}^{h}\right)+\int_{\Gamma_{C}} \frac{1}{\gamma}\left[P_{\gamma}\left(\mathbf{v}^{h}\right)\right]_{+} P_{\theta \gamma}\left(\mathbf{w}^{h}\right) d \Gamma,
$$

for all $\mathbf{v}^{h}, \mathbf{w}^{h} \in \mathbf{V}^{h}$, and where $(\cdot, \cdot)_{1, \Omega}$ stands for the scalar product in $\left(H^{1}(\Omega)\right)^{d}$. Note that problem (10) is well-posed if and only if $\mathbf{B}^{h}$ is a one-to-one operator.

Let $\mathbf{v}^{h}, \mathbf{w}^{h} \in \mathbf{V}^{h}$. We have

$$
\begin{aligned}
& \left(\mathbf{B}^{h} \mathbf{v}^{h}-\mathbf{B}^{h} \mathbf{w}^{h}, \mathbf{v}^{h}-\mathbf{w}^{h}\right)_{1, \Omega} \\
& \quad=a\left(\mathbf{v}^{h}-\mathbf{w}^{h}, \mathbf{v}^{h}-\mathbf{w}^{h}\right)-\theta\left\|\gamma^{\frac{1}{2}} \sigma_{n}\left(\mathbf{v}^{h}-\mathbf{w}^{h}\right)\right\|_{0, \Gamma_{C}}^{2} \\
& \quad+\int_{\Gamma_{C}} \frac{1}{\gamma}\left(\left[P_{\gamma}\left(\mathbf{v}^{h}\right)\right]_{+}-\left[P_{\gamma}\left(\mathbf{w}^{h}\right)\right]_{+}\right)\left(v_{n}^{h}-w_{n}^{h}-\theta \gamma \sigma_{n}\left(\mathbf{v}^{h}-\mathbf{w}^{h}\right)\right) d \Gamma \\
& \quad=a\left(\mathbf{v}^{h}-\mathbf{w}^{h}, \mathbf{v}^{h}-\mathbf{w}^{h}\right)-\theta\left\|\gamma^{\frac{1}{2}} \sigma_{n}\left(\mathbf{v}^{h}-\mathbf{w}^{h}\right)\right\|_{0, \Gamma_{C}}^{2} \\
& \quad+\int_{\Gamma_{C}} \frac{1}{\gamma}\left(\left[P_{\gamma}\left(\mathbf{v}^{h}\right)\right]_{+}-\left[P_{\gamma}\left(\mathbf{w}^{h}\right)\right]_{+}\right) P_{\gamma}\left(\mathbf{v}^{h}-\mathbf{w}^{h}\right) d \Gamma \\
& \quad+(1-\theta) \int_{\Gamma_{C}} \frac{1}{\gamma}\left(\left[P_{\gamma}\left(\mathbf{v}^{h}\right)\right]_{+}-\left[P_{\gamma}\left(\mathbf{w}^{h}\right)\right]_{+}\right) \gamma \sigma_{n}\left(\mathbf{v}^{h}-\mathbf{w}^{h}\right) d \Gamma .
\end{aligned}
$$


Using (6) in (12) and Cauchy-Schwarz inequality, we get

$$
\begin{aligned}
\left(\mathbf{B}^{h} \mathbf{v}^{h}\right. & \left.-\mathbf{B}^{h} \mathbf{w}^{h}, \mathbf{v}^{h}-\mathbf{w}^{h}\right)_{1, \Omega} \\
\geq & a\left(\mathbf{v}^{h}-\mathbf{w}^{h}, \mathbf{v}^{h}-\mathbf{w}^{h}\right)-\theta\left\|\gamma^{\frac{1}{2}} \sigma_{n}\left(\mathbf{v}^{h}-\mathbf{w}^{h}\right)\right\|_{0, \Gamma_{C}}^{2} \\
& +\left\|\gamma^{-\frac{1}{2}}\left(\left[P_{\gamma}\left(\mathbf{v}^{h}\right)\right]_{+}-\left[P_{\gamma}\left(\mathbf{w}^{h}\right)\right]_{+}\right)\right\|_{0, \Gamma_{C}}^{2} \\
& -|1-\theta|\left\|\gamma^{-\frac{1}{2}}\left(\left[P_{\gamma}\left(\mathbf{v}^{h}\right)\right]_{+}-\left[P_{\gamma}\left(\mathbf{w}^{h}\right)\right]_{+}\right)\right\|_{0, \Gamma_{C}}\left\|\gamma^{\frac{1}{2}} \sigma_{n}\left(\mathbf{v}^{h}-\mathbf{w}^{h}\right)\right\|_{0, \Gamma_{C}} .
\end{aligned}
$$

If $\theta=1$, we use the coercivity of $a(\cdot, \cdot)$ and the property (11) in the previous expression (13). Therefore

$$
\begin{aligned}
&\left(\mathbf{B}^{h} \mathbf{v}^{h}-\mathbf{B}^{h} \mathbf{w}^{h}, \mathbf{v}^{h}-\mathbf{w}^{h}\right)_{1, \Omega} \\
& \quad \geq a\left(\mathbf{v}^{h}-\mathbf{w}^{h}, \mathbf{v}^{h}-\mathbf{w}^{h}\right)-\left\|\gamma^{\frac{1}{2}} \sigma_{n}\left(\mathbf{v}^{h}-\mathbf{w}^{h}\right)\right\|_{0, \Gamma_{C}}^{2} \\
& \quad \geq C\left\|\mathbf{v}^{h}-\mathbf{w}^{h}\right\|_{1, \Omega}^{2},
\end{aligned}
$$

when $\gamma_{0}$ is chosen sufficiently small. This corresponds to the symmetric version already studied in 9 .

We now suppose that $\theta \neq 1$. Let $\beta>0$. Applying Young's inequality in (13) yields:

$$
\begin{aligned}
\left(\mathbf{B}^{h} \mathbf{v}^{h}-\right. & \left.\mathbf{B}^{h} \mathbf{w}^{h}, \mathbf{v}^{h}-\mathbf{w}^{h}\right)_{1, \Omega} \\
\geq & a\left(\mathbf{v}^{h}-\mathbf{w}^{h}, \mathbf{v}^{h}-\mathbf{w}^{h}\right)-\theta\left\|\gamma^{\frac{1}{2}} \sigma_{n}\left(\mathbf{v}^{h}-\mathbf{w}^{h}\right)\right\|_{0, \Gamma_{C}}^{2} \\
& +\left\|\gamma^{-\frac{1}{2}}\left(\left[P_{\gamma}\left(\mathbf{v}^{h}\right)\right]_{+}-\left[P_{\gamma}\left(\mathbf{w}^{h}\right)\right]_{+}\right)\right\|_{0, \Gamma_{C}}^{2} \\
& -\frac{|1-\theta|}{2 \beta}\left\|\gamma^{-\frac{1}{2}}\left(\left[P_{\gamma}\left(\mathbf{v}^{h}\right)\right]_{+}-\left[P_{\gamma}\left(\mathbf{w}^{h}\right)\right]_{+}\right)\right\|_{0, \Gamma_{C}}^{2} \\
& -\frac{|1-\theta| \beta}{2}\left\|\gamma^{\frac{1}{2}} \sigma_{n}\left(\mathbf{v}^{h}-\mathbf{w}^{h}\right)\right\|_{0, \Gamma_{C}}^{2} \\
= & a\left(\mathbf{v}^{h}-\mathbf{w}^{h}, \mathbf{v}^{h}-\mathbf{w}^{h}\right)-\left(\theta+\frac{|1-\theta| \beta}{2}\right)\left\|\gamma^{\frac{1}{2}} \sigma_{n}\left(\mathbf{v}^{h}-\mathbf{w}^{h}\right)\right\|_{0, \Gamma_{C}}^{2} \\
& +\left(1-\frac{|1-\theta|}{2 \beta}\right)\left\|\gamma^{-\frac{1}{2}}\left(\left[P_{\gamma}\left(\mathbf{v}^{h}\right)\right]_{+}-\left[P_{\gamma}\left(\mathbf{w}^{h}\right)\right]_{+}\right)\right\|_{0, \Gamma_{C}}^{2} \cdot
\end{aligned}
$$

Choosing $\beta=|1-\theta| / 2$ in (15), we get:

$$
\begin{aligned}
& \left(\mathbf{B}^{h} \mathbf{v}^{h}-\mathbf{B}^{h} \mathbf{w}^{h}, \mathbf{v}^{h}-\mathbf{w}^{h}\right)_{1, \Omega} \geq a\left(\mathbf{v}^{h}-\mathbf{w}^{h}, \mathbf{v}^{h}-\mathbf{w}^{h}\right) \\
& \quad-\frac{1}{4}(1+\theta)^{2}\left\|\gamma^{\frac{1}{2}} \sigma_{n}\left(\mathbf{v}^{h}-\mathbf{w}^{h}\right)\right\|_{0, \Gamma_{C}}^{2} \\
& \quad \geq C\left\|\mathbf{v}^{h}-\mathbf{w}^{h}\right\|_{1, \Omega}^{2},
\end{aligned}
$$

when $\theta \neq-1$ and $\gamma_{0}$ sufficiently small, or when $\theta=-1$. Note that in the latter case we do not need the smallness assumption on $\gamma_{0}$.

Next, let us show that $\mathbf{B}^{h}$ is also hemicontinuous. Since $\mathbf{V}^{h}$ is a vector space, it is sufficient to show that

$$
[0,1] \ni t \mapsto \varphi(t):=\left(\mathbf{B}^{h}\left(\mathbf{v}^{h}-t \mathbf{w}^{h}\right), \mathbf{w}^{h}\right)_{1, \Omega} \in \mathbb{R}
$$


is a continuous real function, for all $\mathbf{v}^{h}, \mathbf{w}^{h} \in \mathbf{V}^{h}$. Let $s, t \in[0,1]$ and we have

$$
\begin{aligned}
& |\varphi(t)-\varphi(s)| \\
= & \left|\left(\mathbf{B}^{h}\left(\mathbf{v}^{h}-t \mathbf{w}^{h}\right)-\mathbf{B}^{h}\left(\mathbf{v}^{h}-s \mathbf{w}^{h}\right), \mathbf{w}^{h}\right)_{1, \Omega}\right| \\
= & \left|A_{\theta \gamma}\left((s-t) \mathbf{w}^{h}, \mathbf{w}^{h}\right)+\int_{\Gamma_{C}} \frac{1}{\gamma}\left(\left[P_{\gamma}\left(\mathbf{v}^{h}-t \mathbf{w}^{h}\right)\right]_{+}-\left[P_{\gamma}\left(\mathbf{v}^{h}-s \mathbf{w}^{h}\right)\right]_{+}\right) P_{\theta \gamma}\left(\mathbf{w}^{h}\right) d \Gamma\right| \\
\leq & |s-t| A_{\theta \gamma}\left(\mathbf{w}^{h}, \mathbf{w}^{h}\right)+\int_{\Gamma_{C}} \frac{1}{\gamma}\left|\left[P_{\gamma}\left(\mathbf{v}^{h}-t \mathbf{w}^{h}\right)\right]_{+}-\left[P_{\gamma}\left(\mathbf{v}^{h}-s \mathbf{w}^{h}\right)\right]_{+}\right|\left|P_{\theta \gamma}\left(\mathbf{w}^{h}\right)\right| d \Gamma .
\end{aligned}
$$

With help of the bound $\left|[a]_{+}-[b]_{+}\right| \leq|a-b|$, for all $a, b \in \mathbb{R}$, and using the linearity of $P_{\gamma}$, we deduce that

$$
\begin{aligned}
\int_{\Gamma_{C}} \frac{1}{\gamma} & \left|\left[P_{\gamma}\left(\mathbf{v}^{h}-t \mathbf{w}^{h}\right)\right]_{+}-\left[P_{\gamma}\left(\mathbf{v}^{h}-s \mathbf{w}^{h}\right)\right]_{+}\right|\left|P_{\theta \gamma}\left(\mathbf{w}^{h}\right)\right| d \Gamma \\
& \leq \int_{\Gamma_{C}} \frac{1}{\gamma}\left|P_{\gamma}\left(\mathbf{v}^{h}-t \mathbf{w}^{h}\right)-P_{\gamma}\left(\mathbf{v}^{h}-s \mathbf{w}^{h}\right)\right|\left|P_{\theta \gamma}\left(\mathbf{w}^{h}\right)\right| d \Gamma \\
& =\int_{\Gamma_{C}} \frac{1}{\gamma}\left|(s-t) P_{\gamma}\left(\mathbf{w}^{h}\right)\right|\left|P_{\theta \gamma}\left(\mathbf{w}^{h}\right)\right| d \Gamma .
\end{aligned}
$$

It gives that

$$
|\varphi(t)-\varphi(s)| \leq|s-t|\left(A_{\theta \gamma}\left(\mathbf{w}^{h}, \mathbf{w}^{h}\right)+\int_{\Gamma_{C}} \frac{1}{\gamma}\left|P_{\gamma}\left(\mathbf{w}^{h}\right)\right|\left|P_{\theta \gamma}\left(\mathbf{w}^{h}\right)\right| d \Gamma\right),
$$

which means that $\varphi$ is Lipschitz, so that $\mathbf{B}^{h}$ is hemicontinuous. Since properties (14), (16) also hold, we finally apply Corollary 15 (p. 126) of 8 to conclude that $\mathbf{B}^{h}$ is a one-to-one operator. This ends the proof.

Remark 3.5. When $\gamma_{0}$ is large and $\theta \neq-1$ we can neither conclude to uniqueness nor to existence of a solution. In Appendix B, we show some simple explicit examples of non-existence and non-uniqueness of solutions.

3.3. A priori error analysis. Our Nitsche-based method (10) converges in an optimal way as the mesh parameter $h$ vanishes. This is proved in the following theorems. First, we establish an abstract error estimate.

Theorem 3.6. Suppose that the solution $\mathbf{u}$ of Problem (3) belongs to $\left(H^{\frac{3}{2}+\nu}(\Omega)\right)^{d}$ with $\nu>0$ and $d=2$ or $d=3$.

1. Let $\theta \in \mathbb{R}$. Suppose that the parameter $\gamma_{0}>0$ is sufficiently small. Then, the solution $\mathbf{u}^{h}$ of Problem (10) satisfies the following abstract error estimate:

$$
\begin{aligned}
& \left\|\mathbf{u}-\mathbf{u}^{h}\right\|_{1, \Omega}+\left\|\gamma^{\frac{1}{2}}\left(\sigma_{n}(\mathbf{u})+\frac{1}{\gamma}\left[P_{\gamma}\left(\mathbf{u}^{h}\right)\right]_{+}\right)\right\|_{0, \Gamma_{C}} \\
& \quad \leq C \inf _{\mathbf{v}^{h} \in \mathbf{V}^{h}}\left(\left\|\mathbf{u}-\mathbf{v}^{h}\right\|_{1, \Omega}+\left\|\gamma^{-\frac{1}{2}}\left(u_{n}-v_{n}^{h}\right)\right\|_{0, \Gamma_{C}}+\left\|\gamma^{\frac{1}{2}} \sigma_{n}\left(\mathbf{u}-\mathbf{v}^{h}\right)\right\|_{0, \Gamma_{C}}\right),
\end{aligned}
$$

where $C$ is a positive constant, independent of $h, \mathbf{u}$ and $\gamma_{0}$.

2. Set $\theta=-1$. Then for all values of $\gamma_{0}>0$, the solution $\mathbf{u}^{h}$ of problem (10) satisfies the abstract error estimate (17) where $C$ is a positive constant, dependent on $\gamma_{0}$ but still independent of $h$ and $\mathbf{u}$. 
Proof. Let $\mathbf{v}^{h} \in \mathbf{V}^{h}$. We first use the $\mathbf{V}$-ellipticity and the continuity of $a(\cdot, \cdot)$, as well as Young's inequality, to obtain

$$
\begin{aligned}
\alpha\left\|\mathbf{u}-\mathbf{u}^{h}\right\|_{1, \Omega}^{2} & \leq a\left(\mathbf{u}-\mathbf{u}^{h}, \mathbf{u}-\mathbf{u}^{h}\right) \\
& =a\left(\mathbf{u}-\mathbf{u}^{h},\left(\mathbf{u}-\mathbf{v}^{h}\right)+\left(\mathbf{v}^{h}-\mathbf{u}^{h}\right)\right) \\
& \leq C\left\|\mathbf{u}-\mathbf{u}^{h}\right\|_{1, \Omega}\left\|\mathbf{u}-\mathbf{v}^{h}\right\|_{1, \Omega}+a\left(\mathbf{u}-\mathbf{u}^{h}, \mathbf{v}^{h}-\mathbf{u}^{h}\right) \\
& \leq \frac{\alpha}{2}\left\|\mathbf{u}-\mathbf{u}^{h}\right\|_{1, \Omega}^{2}+\frac{C^{2}}{2 \alpha}\left\|\mathbf{u}-\mathbf{v}^{h}\right\|_{1, \Omega}^{2}+a\left(\mathbf{u}, \mathbf{v}^{h}-\mathbf{u}^{h}\right)-a\left(\mathbf{u}^{h}, \mathbf{v}^{h}-\mathbf{u}^{h}\right),
\end{aligned}
$$

where $\alpha>0$ is the ellipticity constant of $a(.,$.$) . We can transform the term a\left(\mathbf{u}, \mathbf{v}^{h}\right.$ $\left.\mathbf{u}^{h}\right)-a\left(\mathbf{u}^{h}, \mathbf{v}^{h}-\mathbf{u}^{h}\right)$ since $\mathbf{u}$ solves (3), $\mathbf{u}^{h}$ solves (10) and using Lemma 3.1, which yields

$$
\begin{aligned}
\frac{\alpha}{2}\left\|\mathbf{u}-\mathbf{u}^{h}\right\|_{1, \Omega}^{2} \leq & \frac{C^{2}}{2 \alpha}\left\|\mathbf{u}-\mathbf{v}^{h}\right\|_{1, \Omega}^{2}-\theta \int_{\Gamma_{C}} \gamma \sigma_{n}\left(\mathbf{u}^{h}-\mathbf{u}\right) \sigma_{n}\left(\mathbf{v}^{h}-\mathbf{u}^{h}\right) d \Gamma \\
& +\int_{\Gamma_{C}} \frac{1}{\gamma}\left(\left[P_{\gamma}\left(\mathbf{u}^{h}\right)\right]_{+}-\left[P_{\gamma}(\mathbf{u})\right]_{+}\right) P_{\theta \gamma}\left(\mathbf{v}^{h}-\mathbf{u}^{h}\right) d \Gamma .
\end{aligned}
$$

The first integral term in (18) is bounded as

$$
\begin{aligned}
& -\theta \int_{\Gamma_{C}} \gamma \sigma_{n}\left(\mathbf{u}^{h}-\mathbf{u}\right) \sigma_{n}\left(\mathbf{v}^{h}-\mathbf{u}^{h}\right) d \Gamma \\
= & \theta \int_{\Gamma_{C}} \gamma \sigma_{n}\left(\mathbf{v}^{h}-\mathbf{u}^{h}\right) \sigma_{n}\left(\mathbf{v}^{h}-\mathbf{u}^{h}\right) d \Gamma-\theta \int_{\Gamma_{C}} \gamma \sigma_{n}\left(\mathbf{v}^{h}-\mathbf{u}\right) \sigma_{n}\left(\mathbf{v}^{h}-\mathbf{u}^{h}\right) d \Gamma \\
\leq & \theta\left\|\gamma^{\frac{1}{2}} \sigma_{n}\left(\mathbf{v}^{h}-\mathbf{u}^{h}\right)\right\|_{0, \Gamma_{C}}^{2}+|\theta|\left\|\gamma^{\frac{1}{2}} \sigma_{n}\left(\mathbf{v}^{h}-\mathbf{u}\right)\right\|_{0, \Gamma_{C}}\left\|\gamma^{\frac{1}{2}} \sigma_{n}\left(\mathbf{v}^{h}-\mathbf{u}^{h}\right)\right\|_{0, \Gamma_{C}} \\
\leq & \frac{\beta_{1} \theta^{2}}{2}\left\|\gamma^{\frac{1}{2}} \sigma_{n}\left(\mathbf{v}^{h}-\mathbf{u}\right)\right\|_{0, \Gamma_{C}}^{2}+\left(\frac{1}{2 \beta_{1}}+\theta\right)\left\|\gamma^{\frac{1}{2}} \sigma_{n}\left(\mathbf{v}^{h}-\mathbf{u}^{h}\right)\right\|_{0, \Gamma_{C}}^{2},
\end{aligned}
$$

with $\beta_{1}>0$. The second integral term in (18) is considered next:

$$
\begin{aligned}
\int_{\Gamma_{C}} \frac{1}{\gamma}( & {\left.\left[P_{\gamma}\left(\mathbf{u}^{h}\right)\right]_{+}-\left[P_{\gamma}(\mathbf{u})\right]_{+}\right) P_{\theta \gamma}\left(\mathbf{v}^{h}-\mathbf{u}^{h}\right) d \Gamma } \\
= & \int_{\Gamma_{C}} \frac{1}{\gamma}\left(\left[P_{\gamma}\left(\mathbf{u}^{h}\right)\right]_{+}-\left[P_{\gamma}(\mathbf{u})\right]_{+}\right) P_{\gamma}\left(\mathbf{v}^{h}-\mathbf{u}\right) d \Gamma \\
& +\int_{\Gamma_{C}} \frac{1}{\gamma}\left(\left[P_{\gamma}\left(\mathbf{u}^{h}\right)\right]_{+}-\left[P_{\gamma}(\mathbf{u})\right]_{+}\right) P_{\gamma}\left(\mathbf{u}-\mathbf{u}^{h}\right) d \Gamma \\
& +\int_{\Gamma_{C}} \frac{1}{\gamma}\left(\left[P_{\gamma}\left(\mathbf{u}^{h}\right)\right]_{+}-\left[P_{\gamma}(\mathbf{u})\right]_{+}\right) \gamma(1-\theta) \sigma_{n}\left(\mathbf{v}^{h}-\mathbf{u}^{h}\right) d \Gamma
\end{aligned}
$$

Using the bound $\left([a]_{+}-[b]_{+}\right)(b-a) \leq-\left([a]_{+}-[b]_{+}\right)^{2}$ (see (6]) $)$ and two times Cauchy-Schwarz and Young's inequalities in (20) we obtain

$$
\begin{aligned}
\int_{\Gamma_{C}} \frac{1}{\gamma}\left(\left[P_{\gamma}\left(\mathbf{u}^{h}\right)\right]_{+}-\left[P_{\gamma}(\mathbf{u})\right]_{+}\right) P_{\theta \gamma}\left(\mathbf{v}^{h}-\mathbf{u}^{h}\right) d \Gamma \\
\leq \frac{1}{2 \beta_{2}}\left\|\gamma^{\frac{1}{2}}\left(\sigma_{n}(\mathbf{u})+\frac{1}{\gamma}\left[P_{\gamma}\left(\mathbf{u}^{h}\right)\right]_{+}\right)\right\|_{0, \Gamma_{C}}^{2}+\frac{\beta_{2}}{2}\left\|\gamma^{-\frac{1}{2}} P_{\gamma}\left(\mathbf{v}^{h}-\mathbf{u}\right)\right\|_{0, \Gamma_{C}}^{2} \\
\quad-\left\|\gamma^{\frac{1}{2}}\left(\sigma_{n}(\mathbf{u})+\frac{1}{\gamma}\left[P_{\gamma}\left(\mathbf{u}^{h}\right)\right]_{+}\right)\right\|_{0, \Gamma_{C}}^{2} \\
\quad+\frac{|1-\theta|}{2 \beta_{3}}\left\|\gamma^{\frac{1}{2}}\left(\sigma_{n}(\mathbf{u})+\frac{1}{\gamma}\left[P_{\gamma}\left(\mathbf{u}^{h}\right)\right]_{+}\right)\right\|_{0, \Gamma_{C}}^{2}+\frac{|1-\theta| \beta_{3}}{2}\left\|\gamma^{\frac{1}{2}} \sigma_{n}\left(\mathbf{v}^{h}-\mathbf{u}^{h}\right)\right\|_{0, \Gamma_{C}}^{2},
\end{aligned}
$$


with $\beta_{2}>0$ and $\beta_{3}>0$. Noting that

$$
\left\|\gamma^{-\frac{1}{2}} P_{\gamma}\left(\mathbf{v}^{h}-\mathbf{u}\right)\right\|_{0, \Gamma_{C}}^{2} \leq 2\left\|\gamma^{-\frac{1}{2}}\left(u_{n}-v_{n}^{h}\right)\right\|_{0, \Gamma_{C}}^{2}+2\left\|\gamma^{\frac{1}{2}} \sigma_{n}\left(\mathbf{u}-\mathbf{v}^{h}\right)\right\|_{0, \Gamma_{C}}^{2}
$$

and putting together estimates (19) and (21) in (18) gives

$$
\begin{aligned}
\frac{\alpha}{2}\left\|\mathbf{u}-\mathbf{u}^{h}\right\|_{1, \Omega}^{2} & \leq \frac{C^{2}}{2 \alpha}\left\|\mathbf{u}-\mathbf{v}^{h}\right\|_{1, \Omega}^{2}+\left(\frac{\beta_{1} \theta^{2}}{2}+\beta_{2}\right)\left\|\gamma^{\frac{1}{2}} \sigma_{n}\left(\mathbf{u}-\mathbf{v}^{h}\right)\right\|_{0, \Gamma_{C}}^{2} \\
& +\beta_{2}\left\|\gamma^{-\frac{1}{2}}\left(u_{n}-v_{n}^{h}\right)\right\|_{0, \Gamma_{C}}^{2} \\
& +\left(-1+\frac{1}{2 \beta_{2}}+\frac{|1-\theta|}{2 \beta_{3}}\right)\left\|\gamma^{\frac{1}{2}}\left(\sigma_{n}(\mathbf{u})+\frac{1}{\gamma}\left[P_{\gamma}\left(\mathbf{u}^{h}\right)\right]_{+}\right)\right\|_{0, \Gamma_{C}}^{2} \\
& +\left(\frac{1}{2 \beta_{1}}+\theta+\frac{|1-\theta| \beta_{3}}{2}\right)\left\|\gamma^{\frac{1}{2}} \sigma_{n}\left(\mathbf{v}^{h}-\mathbf{u}^{h}\right)\right\|_{0, \Gamma_{C}}^{2} .
\end{aligned}
$$

The norm term on the discrete normal constraints in (22) is bounded as follows by using (11):

$$
\left\|\gamma^{\frac{1}{2}} \sigma_{n}\left(\mathbf{v}^{h}-\mathbf{u}^{h}\right)\right\|_{0, \Gamma_{C}} \leq C \gamma_{0}^{\frac{1}{2}}\left\|\mathbf{v}^{h}-\mathbf{u}^{h}\right\|_{1, \Omega} \leq C \gamma_{0}^{\frac{1}{2}}\left(\left\|\mathbf{v}^{h}-\mathbf{u}\right\|_{1, \Omega}+\left\|\mathbf{u}^{h}-\mathbf{u}\right\|_{1, \Omega}\right) .
$$

For a fixed $\theta \in \mathbb{R}$ we then choose $\beta_{2}$ and $\beta_{3}$ large enough such that

$$
-1+\frac{1}{2 \beta_{2}}+\frac{|1-\theta|}{2 \beta_{3}}<-\frac{1}{2} .
$$

Then choosing $\gamma_{0}$ small enough in (23) and putting the estimate in (22) establishes the first statement of theorem.

We now consider separately the case $\theta=-1$ for which (22) becomes:

$$
\begin{aligned}
\frac{\alpha}{2}\left\|\mathbf{u}-\mathbf{u}^{h}\right\|_{1, \Omega}^{2} & \leq \frac{C^{2}}{2 \alpha}\left\|\mathbf{u}-\mathbf{v}^{h}\right\|_{1, \Omega}^{2} \\
& +\left(\frac{\beta_{1}}{2}+\beta_{2}\right)\left\|\gamma^{\frac{1}{2}} \sigma_{n}\left(\mathbf{u}-\mathbf{v}^{h}\right)\right\|_{0, \Gamma_{C}}^{2}+\beta_{2}\left\|\gamma^{-\frac{1}{2}}\left(u_{n}-v_{n}^{h}\right)\right\|_{0, \Gamma_{C}}^{2} \\
& +\left(-1+\frac{1}{2 \beta_{2}}+\frac{1}{\beta_{3}}\right)\left\|\gamma^{\frac{1}{2}}\left(\sigma_{n}(\mathbf{u})+\frac{1}{\gamma}\left[P_{\gamma}\left(\mathbf{u}^{h}\right)\right]_{+}\right)\right\|_{0, \Gamma_{C}}^{2} \\
& +\left(\frac{1}{2 \beta_{1}}-1+\beta_{3}\right)\left\|\gamma^{\frac{1}{2}} \sigma_{n}\left(\mathbf{v}^{h}-\mathbf{u}^{h}\right)\right\|_{0, \Gamma_{C}}^{2} .
\end{aligned}
$$

Let $\eta>0$ be given. Set $\beta_{1}=1 /(2 \eta), \beta_{2}=1+(1 / \eta)$ and $\beta_{3}=1+\eta$. Therefore (24) becomes

$$
\begin{aligned}
\frac{\alpha}{2}\left\|\mathbf{u}-\mathbf{u}^{h}\right\|_{1, \Omega}^{2} & \leq \frac{C^{2}}{2 \alpha}\left\|\mathbf{u}-\mathbf{v}^{h}\right\|_{1, \Omega}^{2}+\left(\frac{5}{4 \eta}+1\right)\left\|\gamma^{\frac{1}{2}} \sigma_{n}\left(\mathbf{u}-\mathbf{v}^{h}\right)\right\|_{0, \Gamma_{C}}^{2} \\
& +\frac{1+\eta}{\eta}\left\|\gamma^{-\frac{1}{2}}\left(u_{n}-v_{n}^{h}\right)\right\|_{0, \Gamma_{C}}^{2} \\
& -\frac{\eta}{2(1+\eta)}\left\|\gamma^{\frac{1}{2}}\left(\sigma_{n}(\mathbf{u})+\frac{1}{\gamma}\left[P_{\gamma}\left(\mathbf{u}^{h}\right)\right]_{+}\right)\right\|_{0, \Gamma_{C}}^{2}+2 \eta\left\|\gamma^{\frac{1}{2}} \sigma_{n}\left(\mathbf{v}^{h}-\mathbf{u}^{h}\right)\right\|_{0, \Gamma_{C}}^{2} .
\end{aligned}
$$

Let $\gamma_{0}>0$. Set $\eta=\alpha /\left(16 C^{2} \gamma_{0}\right)$ where $C$ is the constant in (23) to conclude the proof of the theorem. 
Remark 3.7. In the case $\theta=-1$, note that the convergence result holds for any value of $\gamma_{0}>0$. However, in that case, the abstract estimate takes the form

$$
\begin{aligned}
\left\|\mathbf{u}-\mathbf{u}^{h}\right\|_{1, \Omega}+\left(\frac{C}{\gamma_{0}+C}\right)^{\frac{1}{2}} \| \gamma^{\frac{1}{2}}\left(\sigma_{n}(\mathbf{u})+\right. & \left.\frac{1}{\gamma}\left[P_{\gamma}\left(\mathbf{u}^{h}\right)\right]_{+}\right) \|_{0, \Gamma_{C}} \\
\leq C\left(1+\gamma_{0}\right)^{\frac{1}{2}} \inf _{\mathbf{v}^{h} \in \mathbf{V}^{h}}\left(\left\|\mathbf{u}-\mathbf{v}^{h}\right\|_{1, \Omega}+\right. & \left\|\gamma^{-\frac{1}{2}}\left(u_{n}-v_{n}^{h}\right)\right\|_{0, \Gamma_{C}} \\
& \left.+\left\|\gamma^{\frac{1}{2}} \sigma_{n}\left(\mathbf{u}-\mathbf{v}^{h}\right)\right\|_{0, \Gamma_{C}}\right),
\end{aligned}
$$

where $C$ is a positive constant, independent of $h, \mathbf{u}$ and $\gamma_{0}$. As a result, the estimation is deteriorated when $\gamma_{0}$ increases, in particular the estimation of the contact stress on $\Gamma_{C}$.

The optimal convergence of the method is stated below.

Theorem 3.8. Suppose that the solution $\mathbf{u}$ to problem (3) belongs to $\left(H^{\frac{3}{2}+\nu}(\Omega)\right)^{d}$ with $0<\nu \leq k-\frac{1}{2} \quad(k=1,2$ is the degree of the finite element method, given in (44) ) and $d=2,3$. When $\theta \neq-1$, suppose in addition that the parameter $\gamma_{0}$ is sufficiently small. The solution $\mathbf{u}^{h}$ of problem (10) satisfies the error estimate

$$
\left\|\mathbf{u}-\mathbf{u}^{h}\right\|_{1, \Omega}+\left\|\gamma^{\frac{1}{2}}\left(\sigma_{n}(\mathbf{u})+\frac{1}{\gamma}\left[P_{\gamma}\left(\mathbf{u}^{h}\right)\right]_{+}\right)\right\|_{0, \Gamma_{C}} \leq C h^{\frac{1}{2}+\nu}\|\mathbf{u}\|_{\frac{3}{2}+\nu, \Omega},
$$

where $C$ is a positive constant, dependent on $\gamma_{0}$ but independent of $h$ and $\mathbf{u}$.

Proof. We need to bound the right terms in estimate (17) and we choose $\mathbf{v}^{h}=\mathcal{I}^{h} \mathbf{u}$ where $\mathcal{I}^{h}$ stands for the Lagrange interpolation operator mapping onto $\mathbf{V}^{h}$. The estimation of the Lagrange interpolation error in the $H^{1}$-norm on a domain $\Omega$ is classical (see, e.g., [7, 13, 14]):

$$
\left\|\mathbf{u}-\mathcal{I}^{h} \mathbf{u}\right\|_{1, \Omega} \leq C h^{\frac{1}{2}+\nu}\|\mathbf{u}\|_{\frac{3}{2}+\nu, \Omega}
$$

for $-\frac{1}{2}<\nu \leq k-\frac{1}{2}$.

The estimation of the term $\left\|\gamma^{-\frac{1}{2}}\left(u_{n}-\left(\mathcal{I}^{h} \mathbf{u}\right)_{n}\right)\right\|_{0, \Gamma_{C}}$ can be done in a very similar manner to [24. Indeed, let $E$ be an edge (resp. a face) of a triangle (resp. tetrahedron) $T \in \mathcal{T}^{h}$ on $\Gamma_{C}$ :

$$
\left\|\gamma^{-\frac{1}{2}}\left(u_{n}-\left(\mathcal{I}^{h} \mathbf{u}\right)_{n}\right)\right\|_{0, E} \leq C h_{T}^{-\frac{1}{2}} h_{T}^{1+\nu}\left\|u_{n}\right\|_{1+\nu, E}=C h^{\frac{1}{2}+\nu}\left\|u_{n}\right\|_{1+\nu, E},
$$

(see [13] for instance). By summation on all the edges (resp. faces) and the trace theorem, gives

$$
\left\|\gamma^{-\frac{1}{2}}\left(u_{n}-\left(\mathcal{I}^{h} \mathbf{u}\right)_{n}\right)\right\|_{0, \Gamma_{C}} \leq C h^{\frac{1}{2}+\nu}\left\|u_{n}\right\|_{1+\nu, \Gamma_{C}} \leq C h^{\frac{1}{2}+\nu}\|\mathbf{u}\|_{\frac{3}{2}+\nu, \Omega}
$$

From Lemma A.1 in Appendix A, (see also [16]), the following estimate also holds:

$$
\left\|\gamma^{\frac{1}{2}} \sigma_{n}\left(\mathbf{u}-\mathcal{I}^{h} \mathbf{u}\right)\right\|_{0, \Gamma_{C}} \leq C h^{\frac{1}{2}+\nu}\|\mathbf{u}\|_{\frac{3}{2}+\nu, \Omega} .
$$

We conclude by inserting the three estimates (26) $-(28)$ into (17).

Remark 3.9. Note that for the theoretical estimation of Theorem 3.8, in the case $\theta \neq-1$, and for $\gamma_{0}$ small, the constant $C$ in the estimate (25) behaves in $O\left(\gamma_{0}^{-\frac{1}{2}}\right)$ (due to the term $\left\|\gamma^{-\frac{1}{2}}\left(u_{n}-v_{n}^{h}\right)\right\|_{0, \Gamma_{C}}$ ), so that taking $\gamma_{0}$ too small theoretically deteriorates the convergence. In the case $\theta=-1$, the same observation can be made, with the additional fact that the convergence is also deteriorated in $O\left(\gamma_{0}\right)$ if $\gamma_{0}$ is too large (see Remark 3.7). However, numerical experiments of Section 
4 reveal that in practice the method is quite robust relatively to the value of $\gamma_{0}$, provided it is sufficiently small when $\theta \neq-1$.

Actually we are not able to obtain estimates for the displacements in the $L^{2}$ norm $\left(\left\|\mathbf{u}-\mathbf{u}^{h}\right\|_{0, \Omega}\right.$ and also $\left.\left\|\mathbf{u}-\mathbf{u}^{h}\right\|_{0, \Gamma_{C}}\right)$ by using the Aubin-Nitsche argument as it is achieved in the linear case (see [16]). Note also that the $L^{2}(\Omega)$-norm estimates for contact problems are not easy to prove and there are to our knowledge only few estimates (see [12]). Nevertheless, we can easily obtain the following error estimate on the weighted $L^{2}\left(\Gamma_{C}\right)$-norm on the normal constraint $\left\|\gamma^{\frac{1}{2}} \sigma_{n}\left(\mathbf{u}-\mathbf{u}^{h}\right)\right\|_{0, \Gamma_{C}}$ (note that $\sigma_{n}\left(\mathbf{u}^{h}\right) \neq-\frac{1}{\gamma}\left[P_{\gamma}\left(\mathbf{u}^{h}\right)\right]_{+}$on $\Gamma_{C}$ contrary to the continuous case).

Corollary 3.10. Suppose that the solution $\mathbf{u}$ to problem (3) belongs to $\left(H^{\frac{3}{2}+\nu}(\Omega)\right)^{d}$ with $0<\nu \leq k-\frac{1}{2} \quad(k=1,2$ is the degree of the finite element method, given in (44) and $d=2,3$. When $\theta \neq-1$, suppose in addition that the parameter $\gamma_{0}$ is sufficiently small. The solution $\mathbf{u}^{h}$ of problem (10) satisfies the error estimate

$$
\left\|\gamma^{\frac{1}{2}} \sigma_{n}\left(\mathbf{u}-\mathbf{u}^{h}\right)\right\|_{0, \Gamma_{C}} \leq C h^{\frac{1}{2}+\nu}\|\mathbf{u}\|_{\frac{3}{2}+\nu, \Omega}
$$

where $C$ is a positive constant, independent of $h$ and $\mathbf{u}$.

Proof. We use (28), (11), (26) and (25) to establish the bound

$$
\begin{aligned}
\left\|\gamma^{\frac{1}{2}} \sigma_{n}\left(\mathbf{u}-\mathbf{u}^{h}\right)\right\|_{0, \Gamma_{C}} & \leq\left\|\gamma^{\frac{1}{2}} \sigma_{n}\left(\mathbf{u}-\mathcal{I}^{h} \mathbf{u}\right)\right\|_{0, \Gamma_{C}}+\left\|\gamma^{\frac{1}{2}} \sigma_{n}\left(\mathcal{I}^{h} \mathbf{u}-\mathbf{u}^{h}\right)\right\|_{0, \Gamma_{C}} \\
& \leq C h^{\frac{1}{2}+\nu}\|\mathbf{u}\|_{\frac{3}{2}+\nu, \Omega}+C \gamma_{0}^{\frac{1}{2}}\left(\left\|\mathcal{I}^{h} \mathbf{u}-\mathbf{u}\right\|_{1, \Omega}+\left\|\mathbf{u}-\mathbf{u}^{h}\right\|_{1, \Omega}\right) \\
& \leq C h^{\frac{1}{2}+\nu}\|\mathbf{u}\|_{\frac{3}{2}+\nu, \Omega}
\end{aligned}
$$

Remark 3.11. Although contact problems are known to be limited in regularity (the regularity $H^{5 / 2}(\Omega)$ is generally the maximal expected regularity for such inequality problems), the use of quadratic finite element methods can be of interest in particular for the most regular solutions lying in $H^{s}(\Omega), 2<s<5 / 2$ (as it is considered in e.g., [5, 23, 34, 38, ).

\section{NumericAl EXPERIMENTS}

In this section, the numerical results of two- and three-dimensional Hertz's contact problems of a disk/sphere with a plane rigid foundation are presented. This slightly exceeds the scope defined in Section 2 since a non-zero initial gap between the elastic solid and the rigid foundation is considered in the computations. Moreover, the tests are performed with $P_{1}$ and isoparametric $P_{2}$ Lagrange finite elements on meshes which are approximations of the real domain.

The finite element library Getfem $++\sqrt{1}$ is used. The discrete contact problem is solved by using a generalized Newton method, which means that problem (10) is derived with respect to $\mathbf{u}^{h}$ to obtain the tangent system. The term "generalized Newton's method" comes from the fact that the positive part $[x]_{+}$is nondifferentiable at $x=0$. However, no special treatment is considered. If a point of non-differentiability is encountered, the tangent system corresponding to one of the two alternatives $(x<0$ or $x>0)$ is chosen arbitrarily. Integrals of the non-linear term on $\Gamma_{C}$ are computed with standard quadrature formulas. Note that the situation where the solution is non-differentiable at an integration point is very rare and

\footnotetext{
${ }^{1}$ See http://download.gna.org/getfem/html/homepage/
} 

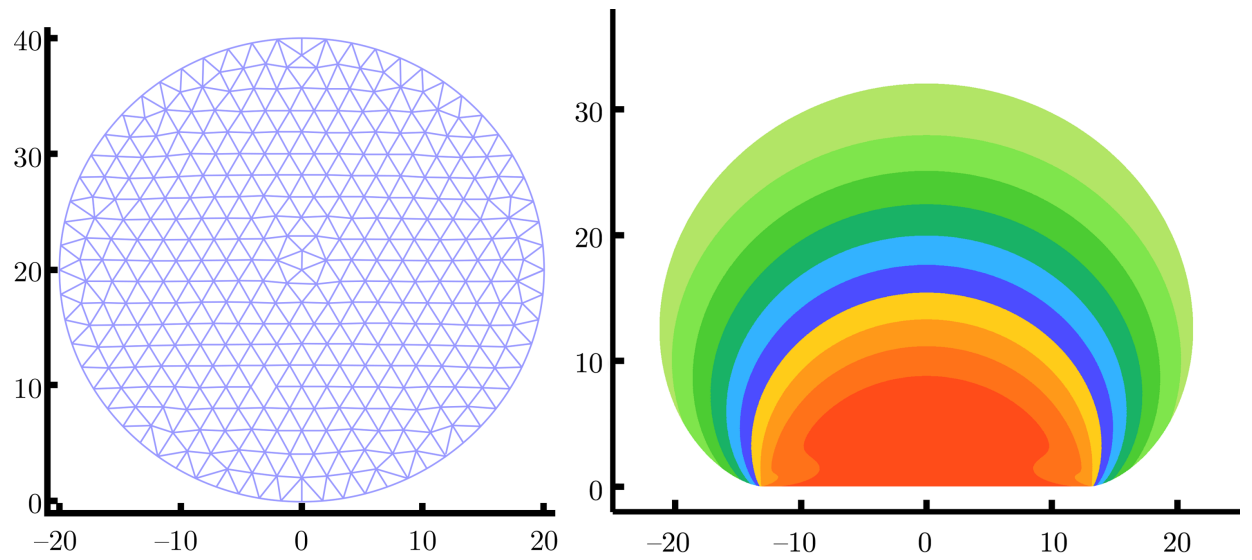

Figure 1. Example of two-dimensional mesh and reference solution (with color plot of the von Mises stress).

corresponds to what is called a "grazing contact" (both $u_{n}=0$ and $\sigma_{n}(\mathbf{u})=0$ ). Further details on the generalized Newton's method applied to contact problems can be found for instance in 35 and the references therein.

4.1. Two-dimensional numerical tests. The numerical situation is represented in Figure 1. A disc of radius $20 \mathrm{~cm}$ is considered with a contact boundary $\Gamma_{C}$ which is restricted to the lower part $(y<20 \mathrm{~cm})$ of the boundary. A homogeneous Neumann condition is applied on the remaining part of the boundary. Since no Dirichlet condition is considered, the problem is not fully coercive. To overcome the non-definiteness coming from the free rigid motions, the horizontal displacement is prescribed to be zero on the two points of coordinates $(0 \mathrm{~cm}, 10 \mathrm{~cm})$ and $(0 \mathrm{~cm}, 30 \mathrm{~cm})$ which blocks the horizontal translation and the rigid rotation. Homogeneous isotropic linear elasticity in plane strain approximation is considered with a Young modulus fixed at $E=25 \mathrm{MPa}$ and a Poisson ratio $P=0.25$. A vertical density of volume forces of $20 \mathrm{MN} / \mathrm{m}^{3}$ is applied.

The error curves when using a $P_{1}$ Lagrange finite element method are shown in Figures 2 3 and 4. The solution for mesh sizes $h=0.5 \mathrm{~cm}, 1 \mathrm{~cm}, 3 \mathrm{~cm}, 4.5 \mathrm{~cm}$ and $h=10 \mathrm{~cm}$ are compared with a reference solution on a very fine mesh $(h=0.15 \mathrm{~cm})$ using quadratic isoparametric finite elements. Moreover, the reference solution is computed with a different discretization of the contact problem (Lagrange multipliers and Alart-Curnier augmented Lagrangian, see [35]). On all the figures, the graph on the left represents the relative $H^{1}(\Omega)$-norm of the error between the computed solution and the reference one and the graph on the right represents the following relative $L^{2}\left(\Gamma_{C}\right)$-norm

$$
\frac{\left\|\gamma^{\frac{1}{2}}\left(\gamma^{-1}\left[P_{\gamma}\left(\mathbf{u}^{h}\right)\right]_{+}-\gamma^{-1}\left[P_{\gamma}\left(\mathbf{u}_{r e f}^{h}\right)\right]_{+}\right)\right\|_{0, \Gamma_{C}}}{\left\|\gamma^{-1}\left[P_{\gamma}\left(\mathbf{u}_{r e f}^{h}\right)\right]_{+}\right\|_{0, \Gamma_{C}}},
$$

where $\mathbf{u}^{h}$ is the discrete solution and $\mathbf{u}_{r e f}^{h}$ the reference solution. The slopes shown in the figures give an approximation of the convergence rate. They correspond to the slopes of the regression lines on a logarithmic scale for the different experiments. 

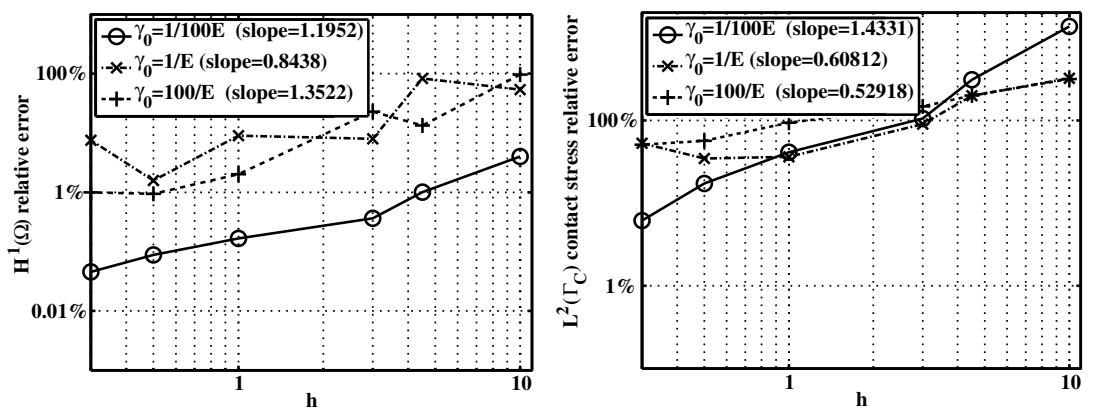

Figure 2 . Error curves in the $2 \mathrm{D}$ case with $P_{1}$ elements and $\theta=1$. Left: relative $H^{1}(\Omega)$-norm on the displacements. Right: relative weighted $L^{2}\left(\Gamma_{C}\right)$-norm on the contact pressure.

Note that $-\frac{1}{\gamma}\left[P_{\gamma}\left(\mathbf{u}^{h}\right)\right]_{+}$is an approximation of the contact stress with a convergence of order 1 (see Theorem 3.8).

Let us recall that the formulation is symmetric if and only if $\theta=1$. The results corresponding to this symmetric case are shown in Figure 2, Optimal convergence is obtained for both $H^{1}(\Omega)$ and weighted $L^{2}\left(\Gamma_{C}\right)$-norms of the error, but only for the smallest value of the parameter $\gamma_{0}\left(\gamma_{0}=1 /(100 E)\right)$. For higher values of $\gamma_{0}$, at least the convergence of the contact stress is non-optimal. This corroborates the theoretical result of Theorem 3.8 for which the optimal rate of convergence is obtained for a sufficiently small $\gamma_{0}$. We also noted that for the largest value of $\gamma_{0}$ (i.e., $\gamma_{0}=100 / E$ ) the convergence of Newton's method is not always achieved (in this case, Newton's method is stopped after 100 iterations). In some experiments, the residual of Newton's method diminishes to a value which is greatly higher than the one we considered for the tests achieving the convergence. Our interpretation is that for large values of $\gamma_{0}$, when coercivity is lost, there might be no solution to the discrete problem.

Now, when $\theta=0$, the error curves are plotted in Figure 3. For this version, which is in a sense simpler to implement than the other ones (since the number of additional terms is lower), Newton's method always converges even for the largest value of $\gamma_{0}$. For this latter value of $\gamma_{0}$ the convergence remains sub-optimal. However, for the intermediate value of $\gamma_{0}\left(\gamma_{0}=1 / E\right)$ the optimal convergence is reached.

Concerning the version with $\theta=-1$, which corresponds to an unconditionally coercive problem, one can see in Figure 4 that optimal convergence is reached for all values of $\gamma_{0}$. Moreover, the smallest error on the contact stress corresponds to the intermediate value of $\gamma_{0}$.

Globally, it is remarkable that the error curves for the smallest value of $\gamma_{0}$ are rather the same for the three values of $\theta$. A strategy to guarantee an optimal convergence is of course to consider a sufficiently small $\gamma_{0}$. However, the price to pay is an ill-conditioned discrete problem. The study presented in 35] for the versions $\theta=1$ and $\theta=0$ shows that Newton's method has important difficulties to converge when $\gamma_{0}$ is small. When symmetry is not required, a better strategy seems to consider the version with $\theta=-1$ or an intermediate value of $\theta=0$ which ensure both a optimal convergence rate and few iterations of Newton's method to converge. 
We have no interpretation of the slight super-convergence noted on most of the error curves. Theoretically, the convergence rates should be close to 1 , since, if we assume that there are only two transition points between effective contact and non-contact, the solution to the continuous problem should be in $\left(H^{s}(\Omega)\right)^{2}$ for all $s<5 / 2$ (see [32]).

The same numerical experiment has been extended to the $P_{2}$ Lagrange isoparametric finite element method. The corresponding error curves are presented in Figures 5, 6 and 17. The results are quite similar compared with the $P_{1}$ Lagrange method. The convergence is even poorer for large values of $\gamma_{0}$ and $\theta=1$. The error levels are smaller compared with the $P_{1}$ method. However, the convergence rates are only slightly better. This comes probably from the regularity of the solution to the continuous problem.
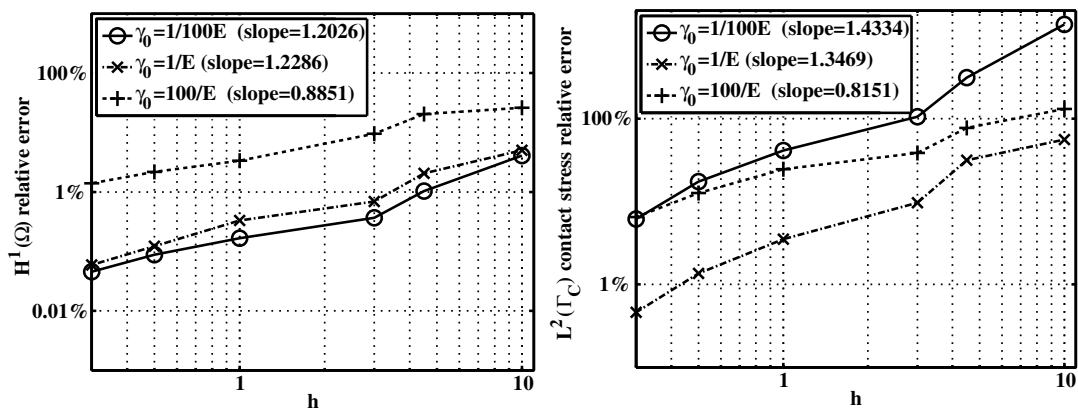

Figure 3 . Error curves in the $2 \mathrm{D}$ case with $P_{1}$ elements and $\theta=0$. Left: relative $H^{1}(\Omega)$-norm on the displacements. Right: relative weighted $L^{2}\left(\Gamma_{C}\right)$-norm on the contact pressure.
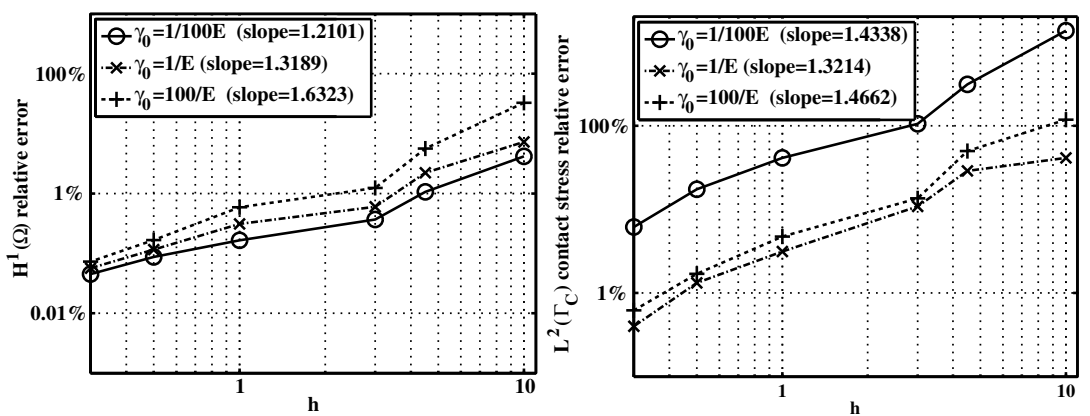

Figure 4. Error curves in the $2 \mathrm{D}$ case with $P_{1}$ elements and $\theta=-1$. Left: relative $H^{1}(\Omega)$-norm on the displacements. Right: relative weighted $L^{2}\left(\Gamma_{C}\right)$-norm on the contact pressure.

4.2. Three-dimensional numerical tests. The three-dimensional tests are similar to the two-dimensional ones. We consider a sphere of radius $20 \mathrm{~cm}$ with mesh sizes $h=3.6 \mathrm{~cm}, 6 \mathrm{~cm}, 11 \mathrm{~cm}, 23 \mathrm{~cm}$ and a $P_{1}$ Lagrange finite element method (an example of a mesh and a reference solution are presented in Figure 8 ). Homogeneous 

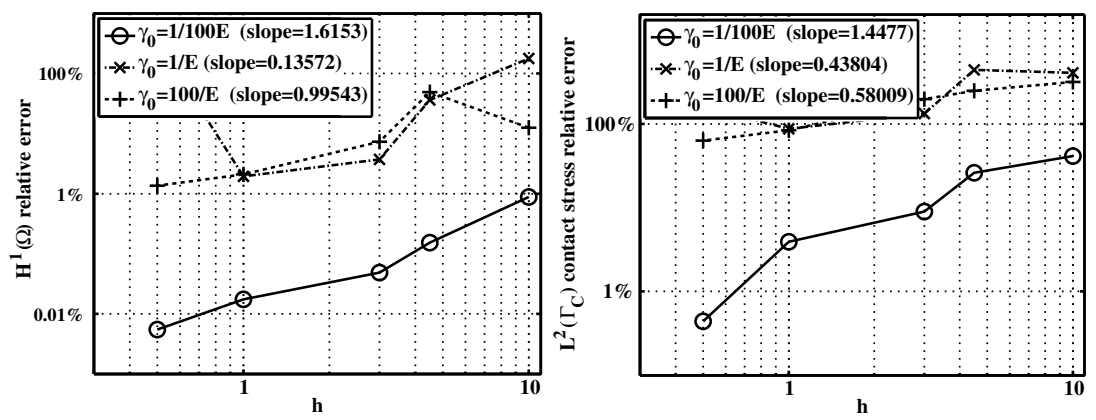

Figure 5. Error curves in the $2 \mathrm{D}$ case with $P_{2}$ elements and $\theta=1$. Left: relative $H^{1}(\Omega)$-norm on the displacements. Right: relative weighted $L^{2}\left(\Gamma_{C}\right)$-norm on the contact pressure.
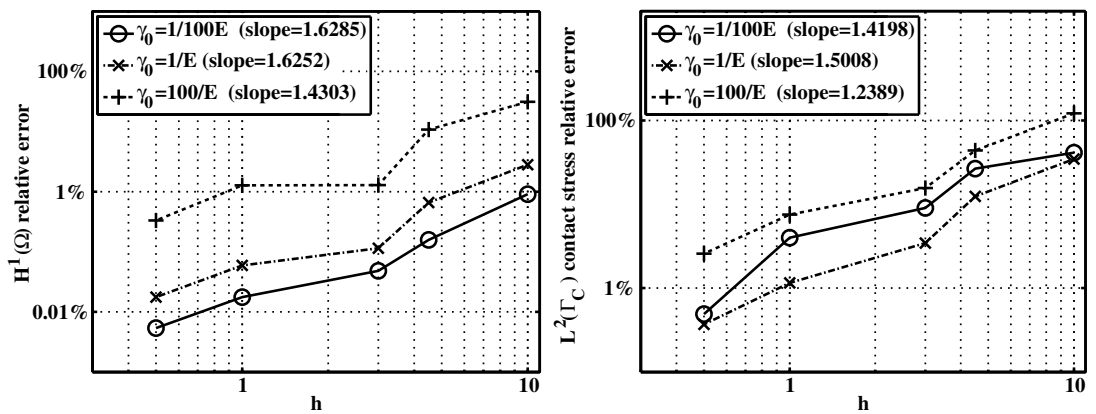

Figure 6 . Error curves in the $2 \mathrm{D}$ case with $P_{2}$ elements and $\theta=0$. Left: relative $H^{1}(\Omega)$-norm on the displacements. Right: relative weighted $L^{2}\left(\Gamma_{C}\right)$-norm on the contact pressure.
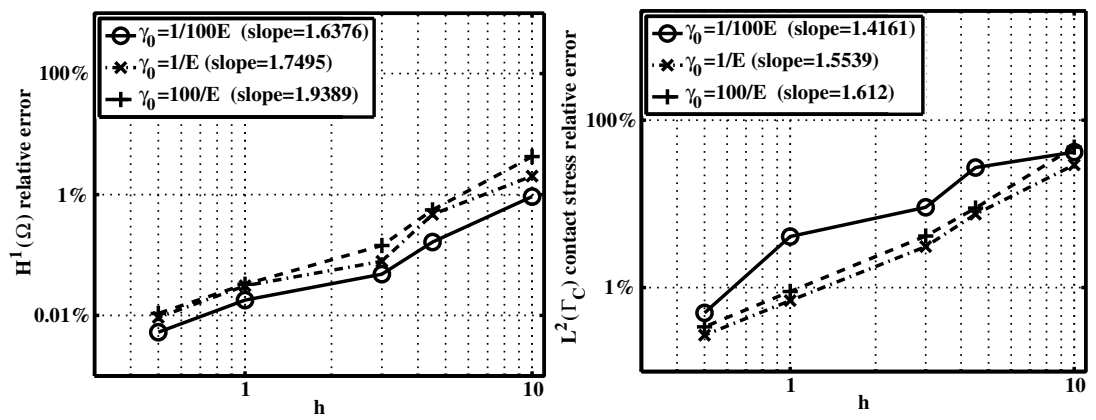

FiguRE 7 . Error curves in the $2 \mathrm{D}$ case with $P_{2}$ elements and $\theta=-1$. Left: relative $H^{1}(\Omega)$-norm on the displacements. Right: relative weighted $L^{2}\left(\Gamma_{C}\right)$-norm on the contact pressure.

isotropic linear elasticity is considered with still a Young modulus $E=25 \mathrm{MPa}$ and a Poisson ratio $P=0.25$. A vertical density of volume forces of $20 \mathrm{MN} / \mathrm{m}^{3}$ is also still considered. Similarly to the two-dimensional case, the horizontal rigid motions 
and the rotations are blocked by prescribing the displacement on specific chosen points. The reference solution is still computed with quadratic isoparametric finite elements on a fine mesh $(h=1 \mathrm{~cm})$ using Lagrange multipliers. The error curves are presented in Figures 9, 10, and 11. Very similar conclusions can be drawn compared with the two-dimensional case. In particular, the method for $\theta=-1$ allows us to obtain an optimal convergence rate for any value of $\gamma_{0}$. However, note that the convergence for large values of $\gamma_{0}$ for $\theta=1$ and $\theta=0$ is even worse compared to the two-dimensional case.

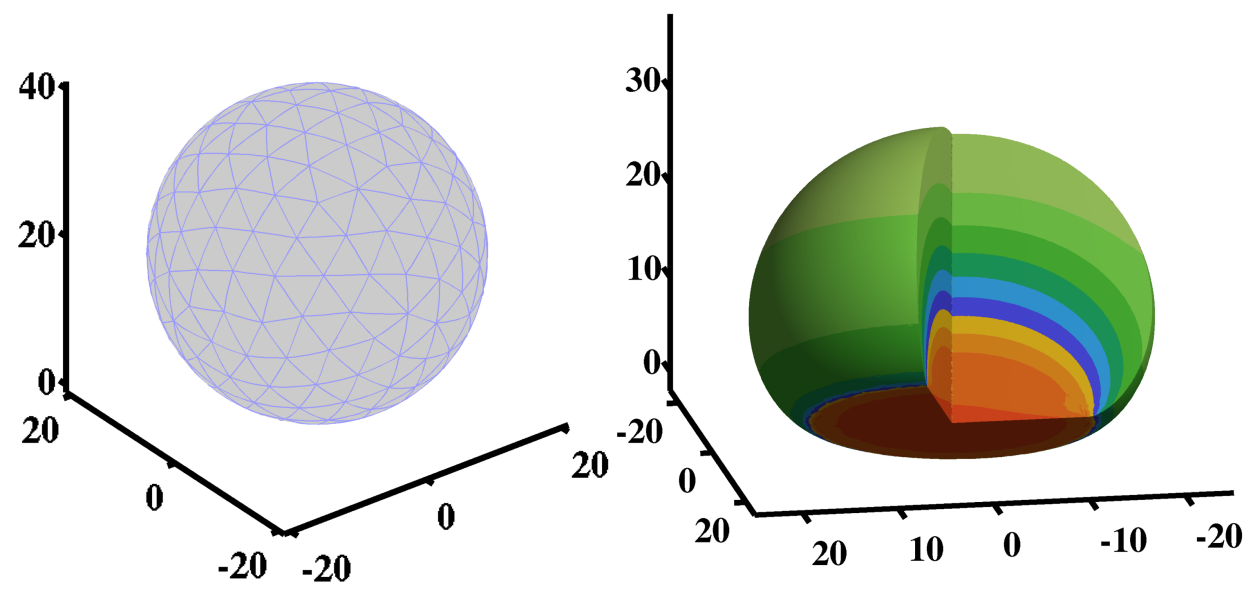

Figure 8. Three-dimensional mesh example and reference solution (sectional view, with color plot of the von Mises stress).
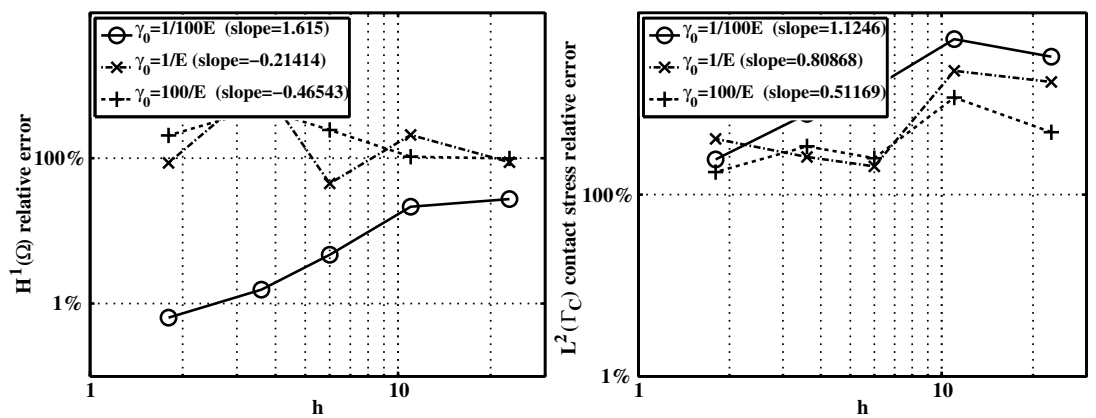

Figure 9. Error curves in the 3D case with $P_{1}$ elements and $\theta=1$. Left: relative $H^{1}(\Omega)$-norm on the displacements. Right: relative weighted $L^{2}\left(\Gamma_{C}\right)$-norm on the contact pressure. 

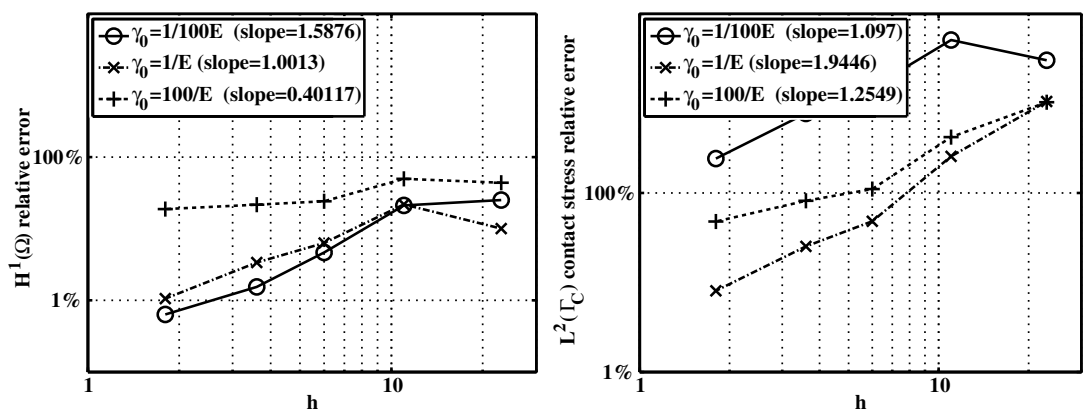

Figure 10. Error curves in the $3 \mathrm{D}$ case with $P_{1}$ elements and $\theta=0$. Left: relative $H^{1}(\Omega)$-norm on the displacements. Right: relative weighted $L^{2}\left(\Gamma_{C}\right)$-norm on the contact pressure.
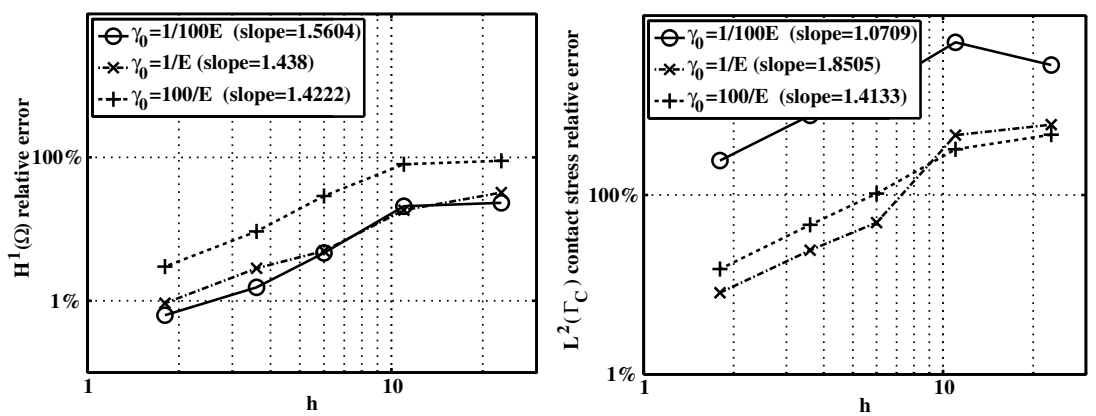

FiguRE 11. Error curves in the $3 \mathrm{D}$ case with $P_{1}$ elements and $\theta=-1$. Left: relative $H^{1}(\Omega)$-norm on the displacements. Right: relative weighted $L^{2}\left(\Gamma_{C}\right)$-norm on the contact pressure.

\section{Conclusion And PeRspectives}

In comparison with the other existing methods for unilateral contact, Nitsche's method has the great advantage that no other unknown than the displacement field is introduced. Indeed, most of the methods are based on a mixed formulation which introduces Lagrange multipliers; see e.g. 39 for a recent review and 35 for a recent numerical comparison of existing methods. From this point of view, Nitsche's method is close to the well-known penalty method in its simplicity, with the advantage of remaining consistent. Moreover, it admits some variants that have interesting properties from the theoretical and/or computational point of view: the variant $\theta=1$ preserves symmetry and has a positive penalty term, the variant $\theta=0$ involves less terms and the variant $\theta=-1$ preserves well-posedness and the convergence irrespectively of the parameter $\gamma_{0}$. For all the variants, i.e., all the values of $\theta$, the discrete problem is well-posed and optimal convergence is achieved if $\gamma_{0}$ is sufficiently small when $\theta \neq-1$. Numerical experiments point out the good convergence properties of Nitsche's formulation when solved with a generalized Newton's method. In particular, the variant $\theta=-1$ presents very good convergence properties, for both small and large values of $\gamma_{0}$. 
Among future directions of work is extension of the method for friction problems, in particular Tresca's friction and then Coulomb's friction.

\section{APPENDIX A: INTERPOLATION ERROR ESTIMATE FOR THE GRADIENT ON THE BOUNDARY}

Lemma A.1. Let $u \in H^{\frac{3}{2}+\nu}(\Omega)$ where $\Omega \subset \mathbb{R}^{d}$ and $0<\nu \leq k-\frac{1}{2} \quad(k=1,2$ is the degree of the finite element method, given in (4)). Set $\Gamma:=\partial \Omega$ and consider an element $T \in \mathcal{T}^{h}$ such that $\Gamma \cap T$ is a face of $T$. Then there exists a positive constant $C$ independent of $T$ and $h$ such that

$$
\left\|\nabla\left(u-\mathcal{I}^{h} u\right)\right\|_{0, \Gamma \cap T} \leq C h_{T}^{\nu}|u|_{\frac{3}{2}+\nu, T}
$$

where $h_{T}$ is the diameter of $T$ and $|u|_{\frac{3}{2}+\nu, T}$ is the usual $H^{\frac{3}{2}+\nu}(T)$ seminorm of $u$.

Proof. We will use a classical scaling argument (see for instance [14, Theorem 1.103]). Let us consider the reference element $\hat{T}$ (which is independent of $T$ and $h_{T}$ ) and the Jacobian matrix $J_{T}$ of the linear geometric transformation from $\hat{T}$ to $T$. Then, due to the regularity of the family of meshes $\mathcal{T}^{h}$, we have

$$
\left|\operatorname{det}\left(J_{T}\right)\right|=\frac{|T|}{|\hat{T}|},\left\|J_{T}\right\| \leq \frac{h_{T}}{\rho_{\hat{T}}},\left\|J_{T}^{-1}\right\| \leq \frac{h_{\hat{T}}}{\rho_{T}},
$$

where $\left\|J_{T}\right\|:=\sup _{\hat{x} \neq 0}\left(\left\|J_{T} \hat{x}\right\| /\|\hat{x}\|\right)$ is the matrix norm associated to the usual euclidean norm in $\mathbb{R}^{d}$ and $|T|,|\hat{T}|$ stand for the areas of $T, \hat{T}$. Using this and the regularity of the mesh, we deduce from a basic calculus

$$
\left\|\nabla\left(u-\mathcal{I}^{h} u\right)\right\|_{0, \Gamma \cap T} \leq C h_{T}^{\frac{d-3}{2}}\left\|\hat{\nabla}\left(\hat{u}-\hat{\mathcal{I}}^{h} \hat{u}\right)\right\|_{0, \hat{\Gamma}},
$$

where $\hat{\Gamma}$ is the corresponding face on the reference element, $\hat{\nabla}, \hat{\mathcal{I}}^{h}$ are the gradient and the Lagrange interpolation operator in the reference coordinates, respectively, and $\hat{u}(\hat{x})=u\left(J_{T}(\hat{x})\right)$. Now, we consider the map

$$
\begin{aligned}
\mathscr{F}: H^{\frac{3}{2}+\nu}(\hat{T}) & \rightarrow\left(L^{2}(\hat{\Gamma})\right)^{d}, \\
\hat{u} & \mapsto \hat{\nabla}\left(\hat{u}-\hat{\mathcal{I}}^{h} \hat{u}\right) .
\end{aligned}
$$

From standard trace theorems (see [1]), we deduce that $\mathscr{F}$ is continuous. Using the property $\mathscr{F}(\hat{p})=0$ for all $\hat{p} \in P_{k}(\hat{T})$, we can write

$$
\begin{aligned}
\left\|\hat{\nabla}\left(\hat{u}-\hat{\mathcal{I}}^{h} \hat{u}\right)\right\|_{0, \hat{\Gamma}} & =\|\mathscr{F}(\hat{u}+\hat{p})\|_{0, \hat{\Gamma}} \forall \hat{p} \in P_{k}(\hat{T}), \\
& \leq\|\mathscr{F}\|_{\mathscr{L}\left(H^{\frac{3}{2}+\nu}(\hat{T}),\left(L^{2}(\hat{\Gamma})\right)^{d}\right)}\|\hat{u}+\hat{p}\|_{\frac{3}{2}+\nu, \hat{T}} \forall \hat{p} \in P_{k}(\hat{T}), \\
& \leq C \inf _{\hat{p} \in P_{k}(\hat{T})}\|\hat{u}+\hat{p}\|_{\frac{3}{2}+\nu, \hat{T}} \\
& \leq C|\hat{u}|_{\frac{3}{2}+\nu, \hat{T}} .
\end{aligned}
$$

The last estimate is the application of the extension to fractional order spaces of Deny-Lions lemma. Such an extension can be found for instance in 13. (Theorem 6.1). Now, proceeding to the reverse change of variable, and still using the regularity 
of the mesh and the expression of $|\hat{u}|_{\frac{3}{2}+\nu, \hat{T}}$ given also for instance in [13] we get

$$
\begin{aligned}
\left\|\nabla\left(u-\mathcal{I}^{h} u\right)\right\|_{0, \Gamma \cap T} & \leq C h_{T}^{\frac{d-3}{2}}|\hat{u}|_{\frac{3}{2}+\nu, \hat{T}} \\
& \leq C h_{T}^{\frac{d-3}{2}} h_{T}^{-\frac{d-3-2 \nu}{2}}|u|_{\frac{3}{2}+\nu, T} \\
& =C h_{T}^{\nu}|u|_{\frac{3}{2}+\nu, T}
\end{aligned}
$$

This result can be straightforwardly extended to the vectorial case. The global interpolation estimate on the whole $\Gamma_{C}$ can be obtained by summation on all the faces of elements lying on $\Gamma_{C}$.

\section{ApPendix B: A Simple EXAMPle of NONEXistence \\ AND NONUNIQUENESS OF SOLUTIONS}

We consider the triangle $\Omega$ of vertexes $A=(0,0), B=(\ell, 0)$ and $C=(0, \ell)$. We define $\Gamma_{D}=[B, C], \Gamma_{N}=[A, C], \Gamma_{C}=[A, B]$ and $\left\{X_{1}, X_{2}\right\}$ denotes the canonical orthonormal basis (see Figure 12). We suppose that the volume forces $\mathbf{f}$ are absent and that the surface forces denoted $\mathbf{g}=g_{1} X_{1}+g_{2} X_{2}$ are such that $g_{1}$ and $g_{2}$ are constant on $\Gamma_{N}$.

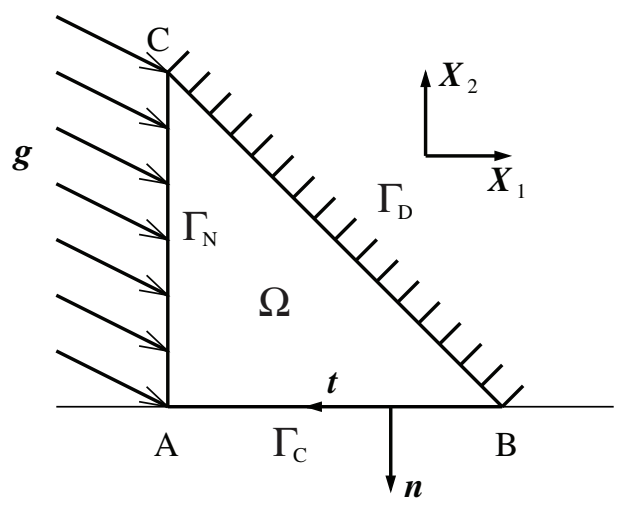

Figure 12. Setting of the problem.

We suppose that $\Omega$ is discretized with a single finite element of degree one. Consequently, the finite element space becomes:

$$
\mathbf{V}^{h}:=\left\{\mathbf{v}^{h}=\left(v_{1}^{h}, v_{2}^{h}\right) \in\left(P_{1}(\Omega)\right)^{2}, \mathbf{v}^{h}=\mathbf{0} \text { on } \Gamma_{D}\right\} .
$$

Clearly, $\mathbf{V}^{h}$ is of dimension two. For $\mathbf{v}^{h} \in \mathbf{V}^{h}$ (resp. $\mathbf{u}^{h} \in \mathbf{V}^{h}$ ), we denote by $\left(V_{T}, V_{N}\right)$ (resp. $\left.\left(U_{T}, U_{N}\right)\right)$ the value of $\mathbf{v}^{h}(A)$ corresponding to the tangential and the normal displacements at point $A$ respectively (in our example, we have $V_{T}=-v_{1}^{h}(A)$ and $\left.V_{N}=-v_{2}^{h}(A)\right)$. Then, for any $\mathbf{v}^{h} \in \mathbf{V}^{h}$, one obtains

$$
\varepsilon\left(\mathbf{v}^{h}\right)=\frac{1}{2 \ell}\left(\begin{array}{cc}
2 V_{T} & V_{T}+V_{N} \\
V_{T}+V_{N} & 2 V_{N}
\end{array}\right) .
$$

We denote by $E$ and $\nu$ the Young modulus and the Poisson ratio or equivalently $\lambda=(E P) /((1-2 P)(1+P))$ and $\mu=E /(2(1+P))$ are the corresponding Lamé 
coefficients. So we get

$$
\boldsymbol{\sigma}\left(\mathbf{v}^{h}\right)=\frac{1}{\ell}\left(\begin{array}{cc}
(\lambda+2 \mu) V_{T}+\lambda V_{N} & \mu\left(V_{T}+V_{N}\right) \\
\mu\left(V_{T}+V_{N}\right) & (\lambda+2 \mu) V_{N}+\lambda V_{T}
\end{array}\right) .
$$

We next consider a very simple case:

$$
E=1, P=0 \text { (i.e., } \lambda=0, \mu=1 / 2), \theta=1, \ell=1, \gamma=1 \text { (i.e., } \gamma_{0}=1 / \sqrt{2} \text { ). }
$$

Therefore

$$
A_{1}\left(\mathbf{u}^{h}, \mathbf{v}^{h}\right)=\frac{3}{4}\left(U_{T} V_{T}+U_{N} V_{N}\right)+\frac{1}{4}\left(U_{T} V_{N}+U_{N} V_{T}\right)-U_{N} V_{N} .
$$

Denoting by $\phi_{N}$ the (normal component) basis function of $\mathbf{V}^{h}\left(\phi_{N}(A)=1, \phi_{N}(B)=\right.$ 0 ), we get

$$
P_{1}\left(\mathbf{v}^{h}\right)=V_{N} \phi_{N}-V_{N} \text { and }\left[P_{1}\left(\mathbf{u}^{h}\right)\right]_{+}=\left(1-\phi_{N}\right)\left(-U_{N}\right)_{+},
$$

so

$$
\int_{\Gamma_{C}} \frac{1}{\gamma}\left[P_{1}\left(\mathbf{u}^{h}\right)\right]_{+} P_{1}\left(\mathbf{v}^{h}\right) d \Gamma=-\frac{1}{3}\left(-U_{N}\right)_{+} V_{N}
$$

Also

$$
L\left(\mathbf{v}^{h}\right)=-\frac{1}{2}\left(g_{1} V_{T}+g_{2} V_{N}\right) .
$$

The discrete problem (10) then consists of finding $\left(U_{T}, U_{N}\right) \in \mathbb{R}^{2}$ such that

$$
\left\{\begin{array}{l}
\frac{3}{2} U_{T}+\frac{1}{2} U_{N}=-g_{1}, \\
\frac{1}{2} U_{T}-\frac{1}{2} U_{N}-\frac{2}{3}\left(-U_{N}\right)_{+}=-g_{2} .
\end{array}\right.
$$

Clearly, a solution of (29) satisfies either $U_{N} \geq 0$ or $U_{N}<0$. We now show that for certain values of $g_{1}$ and $g_{2}$, system (29) admits an infinity of solutions or no solution.

- Suppose that $g_{1}=3 g_{2}$, then there exists an infinity of solutions $\left(U_{T}, U_{N}\right)=$ $\left(-2 g_{1} / 3-x / 3, x\right)$ for any $x \leq 0$.

- Suppose that $g_{1}>3 g_{2}$, then (29) admits no solution.

\section{REFERENCES}

[1] Robert A. Adams, Sobolev spaces, Academic Press [A subsidiary of Harcourt Brace Jovanovich, Publishers], New York-London, 1975. Pure and Applied Mathematics, Vol. 65. MR0450957 (56 \#9247)

[2] A. Curnier and P. Alart, A generalized Newton method for contact problems with friction (English, with French summary), J. Méc. Théor. Appl. 7 (1988), no. suppl. 1, 67-82. MR.988336 (90a:73147)

[3] Douglas N. Arnold, An interior penalty finite element method with discontinuous elements, SIAM J. Numer. Anal. 19 (1982), no. 4, 742-760, DOI 10.1137/0719052. MR664882 (83f:65173)

[4] Roland Becker, Peter Hansbo, and Rolf Stenberg, A finite element method for domain decomposition with non-matching grids, M2AN Math. Model. Numer. Anal. 37 (2003), no. 2, 209-225, DOI 10.1051/m2an:2003023. MR.1991197 (2004e:65129)

[5] Z. Belhachmi and F. Ben Belgacem, Quadratic finite element approximation of the Signorini problem, Math. Comp. 72 (2003), no. 241, 83-104 (electronic), DOI 10.1090/S0025-5718-0101413-2. MR1933319 (2003i:74037)

[6] F. Ben Belgacem and Y. Renard, Hybrid finite element methods for the Signorini problem, Math. Comp. 72 (2003), no. 243, 1117-1145 (electronic), DOI 10.1090/S0025-5718-03-01490X. MR 1972730 (2004d:65139) 
[7] S.-C. Brenner and L.-R. Scott, The Mathematical Theory of Finite Element Methods, Texts in Applied Mathematics, vol. 15, Springer-Verlag, New York, 2007.

[8] Haïm Brezis, Équations et inéquations non linéaires dans les espaces vectoriels en dualité (French), Ann. Inst. Fourier (Grenoble) 18 (1968), no. fasc. 1, 115-175. MR0270222 (42 \#5113)

[9] Franz Chouly and Patrick Hild, A Nitsche-based method for unilateral contact problems: numerical analysis, SIAM J. Numer. Anal. 51 (2013), no. 2, 1295-1307, DOI 10.1137/12088344X. MR3045657

[10] Franz Chouly and Patrick Hild, On convergence of the penalty method for unilateral contact problems, Appl. Numer. Math. 65 (2013), 27-40, DOI 10.1016/j.apnum.2012.10.003. MR3008186

[11] P. G. Ciarlet, Basic error estimates for elliptic problems, Handbook of numerical analysis, Vol. II, Handb. Numer. Anal., II, North-Holland, Amsterdam, 1991, pp. 17-351. MR.1115237

[12] Patrice Coorevits, Patrick Hild, Khalid Lhalouani, and Taoufik Sassi, Mixed finite element methods for unilateral problems: convergence analysis and numerical studies, Math. Comp. 71 (2002), no. 237, 1-25 (electronic), DOI 10.1090/S0025-5718-01-01318-7. MR1862986 (2002g:74043)

[13] Todd Dupont and Ridgway Scott, Polynomial approximation of functions in Sobolev spaces, Math. Comp. 34 (1980), no. 150, 441-463, DOI 10.2307/2006095. MR559195 (81h:65014)

[14] Alexandre Ern and Jean-Luc Guermond, Theory and Practice of Finite Elements, Applied Mathematical Sciences, vol. 159, Springer-Verlag, New York, 2004. MR2050138(2005d:65002)

[15] Gaetano Fichera, Problemi elastostatici con vincoli unilaterali: Il problema di Signorini con ambigue condizioni al contorno (Italian), Atti Accad. Naz. Lincei Mem. Cl. Sci. Fis. Mat. Natur. Sez. I (8) 7 (1963/1964), 91-140. MR0178631 (31 \#2888)

[16] A. Fritz, S. Hüeber, and B. I. Wohlmuth, A comparison of mortar and Nitsche techniques for linear elasticity, Calcolo 41 (2004), no. 3, 115-137, DOI 10.1007/s10092-004-0087-4. MR2199546 (2006j:74031)

[17] Roland Glowinski and Patrick Le Tallec, Augmented Lagrangian and operator-splitting methods in nonlinear mechanics, SIAM Studies in Applied Mathematics, vol. 9, Society for Industrial and Applied Mathematics (SIAM), Philadelphia, PA, 1989. MR1060954 (91f:73038)

[18] Weimin Han and Mircea Sofonea, Quasistatic Contact Problems in Viscoelasticity and Viscoplasticity, AMS/IP Studies in Advanced Mathematics, vol. 30, American Mathematical Society, Providence, RI, 2002. MR 1935666 (2003m:74086)

[19] Anita Hansbo and Peter Hansbo, A finite element method for the simulation of strong and weak discontinuities in solid mechanics, Comput. Methods Appl. Mech. Engrg. 193 (2004), no. 33-35, 3523-3540, DOI 10.1016/j.cma.2003.12.041. MR2075053 (2005b:74116)

[20] J. Haslinger, I. Hlaváček, and J. Nečas, Numerical methods for unilateral problems in solid mechanics, Handb. Numer. Anal., IV, North-Holland, Amsterdam, 1996, pp. 313-485. MR.1422506

[21] Per Heintz and Peter Hansbo, Stabilized Lagrange multiplier methods for bilateral elastic contact with friction, Comput. Methods Appl. Mech. Engrg. 195 (2006), no. 33-36, 43234333, DOI 10.1016/j.cma.2005.09.008. MR2229843 (2007b:74094)

[22] Patrick Hild, Numerical implementation of two nonconforming finite element methods for unilateral contact, Comput. Methods Appl. Mech. Engrg. 184 (2000), no. 1, 99-123, DOI 10.1016/S0045-7825(99)00096-1. MR1752624 (2000k:74058)

[23] Patrick Hild and Patrick Laborde, Quadratic finite element methods for unilateral contact problems, Appl. Numer. Math. 41 (2002), no. 3, 401-421, DOI 10.1016/S0168-9274(01)001246. MR1903172 (2003e:65221)

[24] Patrick Hild and Yves Renard, A stabilized Lagrange multiplier method for the finite element approximation of contact problems in elastostatics, Numer. Math. 115 (2010), no. 1, 101-129, DOI 10.1007/s00211-009-0273-z. MR2594343 (2011a:65405)

[25] Patrick Hild and Yves Renard, An improved a priori error analysis for finite element approximations of Signorini's problem, SIAM J. Numer. Anal. 50 (2012), no. 5, 2400-2419, DOI 10.1137/110857593. MR.3022224

[26] S. Hüeber and B. I. Wohlmuth, An optimal a priori error estimate for nonlinear multibody contact problems, SIAM J. Numer. Anal. 43 (2005), no. 1, 156-173 (electronic), DOI 10.1137/S0036142903436678. MR2177139(2006g:65176) 
[27] N. Kikuchi and J. T. Oden, Contact problems in elasticity: a study of variational inequalities and finite element methods, SIAM Studies in Applied Mathematics, vol. 8, Society for Industrial and Applied Mathematics (SIAM), Philadelphia, PA, 1988. MR.961258 (89j:73097)

[28] Noboru Kikuchi and Young Joon Song, Penalty/finite-element approximations of a class of unilateral problems in linear elasticity, Quart. Appl. Math. 39 (1981/82), no. 1, 1-22. MR613950 (82k:73075)

[29] Patrick Laborde and Yves Renard, Fixed point strategies for elastostatic frictional contact problems, Math. Methods Appl. Sci. 31 (2008), no. 4, 415-441, DOI 10.1002/mma.921. MR2387416 (2009i:74073)

[30] Tod A. Laursen, Computational Contact and Impact Mechanics, Springer-Verlag, Berlin, 2002. Fundamentals of modeling interfacial phenomena in nonlinear finite element analysis. MR.1902698(2003e:74050)

[31] J.-L. Lions, Quelques méthodes de résolution des problèmes aux limites non linéaires (French), Dunod, 1969. MR0259693 (41 \#4326)

[32] Mohand Moussaoui and Khadidja Khodja, Régularité des solutions d'un problème mêlé Dirichlet-Signorini dans un domaine polygonal plan (French, with English and French summaries), Comm. Partial Differential Equations 17 (1992), no. 5-6, 805-826, DOI 10.1080/03605309208820864. MR1177293 (93h:35055)

[33] J. Nitsche, Über ein Variationsprinzip zur Lösung von Dirichlet-Problemen bei Verwendung von Teilräumen, die keinen Randbedingungen unterworfen sind (German), Abh. Math. Sem. Univ. Hamburg 36 (1971), 9-15. MR0341903 (49 \#6649)

[34] A. Popp, B. I. Wohlmuth, M. W. Gee, and W. A. Wall, Dual quadratic mortar finite element methods for $3 D$ finite deformation contact, SIAM J. Sci. Comput. 34 (2012), no. 4, B421B446, DOI 10.1137/110848190. MR2970413

[35] Yves Renard, Generalized Newton's methods for the approximation and resolution of frictional contact problems in elasticity, Comput. Methods Appl. Mech. Engrg. 256 (2013), 38-55, DOI 10.1016/j.cma.2012.12.008. MR3029045

[36] Rolf Stenberg, On some techniques for approximating boundary conditions in the finite element method, J. Comput. Appl. Math. 63 (1995), no. 1-3, 139-148, DOI 10.1016/03770427(95)00057-7. International Symposium on Mathematical Modelling and Computational Methods Modelling 94 (Prague, 1994). MR 1365557 (96j:65129)

[37] Vidar Thomée, Galerkin Finite Element Methods for Parabolic Problems, Springer Series in Computational Mathematics, vol. 25, Springer-Verlag, Berlin, 1997. MR,1479170 (98m:65007)

[38] B. I. Wohlmuth, A. Popp, M. W. Gee, and W. A. Wall, An abstract framework for a priori estimates for contact problems in $3 D$ with quadratic finite elements, Comput. Mech. 49 (2012), no. 6, 735-747, DOI 10.1007/s00466-012-0704-z. MR2935182

[39] Barbara Wohlmuth, Variationally consistent discretization schemes and numerical algorithms for contact problems, Acta Numer. 20 (2011), 569-734, DOI 10.1017/ S0962492911000079. MR2805157 (2012h:74075)

[40] P. Wriggers, Computational contact mechanics, Wiley, 2002.

[41] P. Wriggers and G. Zavarise, A formulation for frictionless contact problems using a weak form introduced by Nitsche, Comput. Mech. 41 (2008), 407-420.

Laboratoire de Mathématiques de BesanÇOn - UMR CNRS 6623, Université de Franche

Comté, 16 route de Gray, 25030 Besançon Cedex, France

E-mail address: franz.chouly@univ-fcomte.fr

Institut de Mathématiques de Toulouse - UMR CNRS 5219, Université Paul Sabatier, 118 route de Narbonne, 31062 Toulouse Cedex 9, France

E-mail address: patrick.hild@math.univ-toulouse.fr

Université de Lyon, CNRS, INSA-Lyon, ICJ UMR5208, LAMCoS UMR5259, F-69621,

Villeurbanne, France

E-mail address: yves.renard@insa-lyon.fr 